\title{
The Electrodynamics of Inhomogeneous Rotating Media and the Abraham and Minkowski Tensors I: General Theory
}

\author{
Shin-itiro Goto, Robin W. Tucker and Timothy J. Walton \\ Department of Physics, Lancaster University, Lancaster and \\ The Cockcroft Institute, Keckwick Lane, Daresbury, UK.
}

\begin{abstract}
This is paper I of a series of two papers, offering a self-contained analysis of the role of electromagnetic stress-energy-momentum tensors in the classical description of continuous polarizable perfectly insulating media. While acknowledging the primary role played by the total stress-energymomentum tensor on spacetime we argue that it is meaningful and useful in the context of covariant constitutive theory to assign preferred status to particular parts of this total tensor, when defined with respect to a particular splitting. The relevance of tensors, associated with the electromagnetic fields that appear in Maxwell's equations for polarizable media, to the forces and torques that they induce has been a matter of some debate since Minkowski, Einstein \& Laub and Abraham considered these issues over a century ago. The notion of a force density that arises from the divergence of these tensors is strictly defined relative to some inertial property of the medium. Consistency with the laws of Newtonian continuum mechanics demands that the total force density on any element of a medium be proportional to the local linear acceleration field of that element in an inertial frame and must also arise as part of the divergence of the total stress-energy-momentum tensor. The fact that, unlike the tensor proposed by Minkowski, the divergence of the Abraham tensor depends explicitly on the local acceleration field of the medium as well as the electromagnetic field, sets it apart from many other terms in the total stress-energy-momentum tensor for a medium.

In this paper we explore how electromagnetic forces or torques on moving media can be defined covariantly in terms of a particular 3-form on those spacetimes that exhibit particular Killing symmetries. It is shown how the drive-forms associated with translational Killing vector fields lead to explicit expressions for the electromagnetic force densities in stationary media subject to the Minkowski constitutive relations and these are compared with other models involving polarizable media in electromagnetic fields that have been considered in the recent literature.
\end{abstract}

\section{Introduction}

The interaction of matter with the electromagnetic field has played a dominant role in the development of our understanding of Nature. In classical Newtonian 
continuum mechanics one is concerned with dynamic processes involving the interaction of (bounded) classical continua with external forces and torques in space (see e.g. [1]). In relativistic continuum mechanics the theory is generalized to incorporate the concept of the spacetime manifold and formulated in terms of tensors on this manifold (see e.g. [2]). In both formulations the histories of configuration variables must be compatible with balance laws associated with possible translational and rotational symmetries of a metric structure and an associated balance law of 'energy'. If electromagnetic fields are involved these laws are supplemented with the macroscopic Maxwell equations in media. As a result of cohesive forces originating at the molecular level the interaction of a material with these (and gravitational influences) results in locally induced strains. These strains determine material stresses that are encoded into various 'stress-tensors'. These in turn describe the local distribution of force and torque densities in the medium. The mathematical structure of such tensors characterizes the response of different materials to external influences. Constitutive relations are relations that are used together with field equations, equations of motion and boundary conditions to fix the dynamic evolution of the independent dynamical variables. If the configuration of the system (open or closed) involves thermodynamic variables the classical laws of thermodynamics can be used to constrain these relations for real media. In the Newtonian formulation one uses the Maxwell and Cauchy stress tensors [3], together with certain equations of state. In the relativistic formulation a primary role is played by the total stress-energy-momentum tensor for the system. The determination of these tensors, together with appropriate constitutive relations for different types of polarizable uncharged media often requires input from experiment. The electromagnetic constitutive properties and the appropriate electromagnetic stressenergy-momentum for light in moving media has been a subject of debate (and possible confusion) for over a century. Difficulties arise in properly accounting for the local nature of the wave-matter interaction in terms of experimentally measurable effects. Even for static electromagnetic fields there are (often nonlinear) subtle interactions that induce changes in shape or volume in deformable media dependent on its thermodynamic state.

There is a considerable body of opinion suggesting that in some sense the choice between different stress-energy-momentum tensors describing interactions of a material medium with an electromagnetic field is a matter of convenience and that different choices simply provide alternative descriptions of the same overall interaction 1$]$ [4, 5, 6, 7]. Since there is no preferred tensor partition of the total stress-energy-momentum tensor into sub-tensors describing the behaviour of interacting sub-systems there can be no unique definition of a stress-energy-momentum tensor describing an interacting subsystem. Thus for electromagnetic fields interacting with an electrically neutral bounded continuum it is always possible to redefine integrated electromagnetic forces and torques on the medium associated with sub-tensors according to taste, particularly if the medium is composed of piecewise inhomogeneous material subsystems or the fields are time dependent. However in order to model the total interacting system the choice of a total stress-energy-momentum tensor is necessary. If one has decided how to model a neutral but polarizable medium in

\footnotetext{
1 The genesis of this idea of classical duality may have its origins in the analogy with wave-particle duality in quantum mechanics.
} 
the absence of externally applied electromagnetic fields then different choices of a stress-energy-momentum tensor for electromagnetic fields in the medium to describe the additional interactions with such fields will inevitable lead in general to different predictions for the interacting system.

Although the laws of classical electromagnetism in the vacuum are firmly established in a relativistic context there is no general agreement on how best to accommodate the dynamics of deformable media as a self consistent theory on spacetime, (see e.g. 8). This makes any rigorous formulation of relativistic continuum mechanics of inhomogeneous dispersive polarizable media interacting with electromagnetic and gravitational fields difficult even if one contemplates using it for systems in non-relativistic motion in some frame of reference.

Despite these shortcomings there has recently been a resurgence of interest in the so-called Abraham-Minkowski controversy and its relevance to the use of either the Abraham or the Minkowski form of stress-energy-momentum tensor in interpreting experiments involving electromagnetic fields in media [9, 10, 11, 12]. Part of this difficulty is no doubt due to the complex nature of material responses to forces in general. From our perspective such experiments are seeking constitutive relations involving particular material systems and the macroscopic Maxwell fields in media. As such it should come as no surprise that different systems might yield different responses particularly if the competing effects of electro- or magneto-striction mentioned above are contributing differently in different experiments.

A number of historic experiments have sought to detect the discriminating Abraham force 13, 14. This is strictly discriminatory only for homogeneous non-dispersive stationary media, so calls into question those experiments that involve media in motion. Indeed the fundamental difference between the forces or torques induced by the divergence of the Abraham and Minkowski electromagnetic stress-energy-momentum tensors is that the former, unlike the latter, can depend explicitly on the acceleration of the medium. From this observation it is our contention that, for any specified electromagnetic constitutive relation, there remain experimental avenues offering new means to discriminate between alternative proposals for the form of the electromagnetic stress-energymomentum tensor in media, despite the attendant inherent material constitutive complexities. In particular we argue that, in principle, the observed dependence of a time-averaged electromagnetic wave-induced torque on the angular speed of a materially isotropic but inhomogeneous uniformly rotating electrically neutral medium could be used to discriminate between the electromagnetic wave interactions described by the Abraham tensor from those described by the symmetrized version of the tensor introduced by Minkowski, and possibly those proposed by others.

It is therefore of interest to calculate how such a torque depends on geometric properties of a cylindrical insulator, its speed of rotation and its electromagnetic constitutive properties. Since the medium will be assumed electrically polarizable and magnetizable (but non-conducting) one is immediately confronted with a number of subtleties associated with the form of this tensor and the material constitutive properties of the medium. These issues have a bearing on how one formulates the classical electromagnetic force on a macroscopic body particularly one that is accelerating. Since this has led to a number of related questions in the recent literature 15, 16, 17, this article attempts to make explicit our perspective. After reviewing approaches in the Newtonian framework the use of 
a fully covariant special relativistic framework is advocated. The essentials of this formulation are described below which should be read in conjunction with the expository material on electromagnetic theory in the language of differential forms contained in the appendix. In the following paper, applications to rotating media are discussed in this context.

\section{Points of Departure}

In order to motivate our method of analysis leading to a computation of the electromagnetic torque on an inhomogeneous rotating uncharged insulator, it is useful to place our methodology in the context of the recent literature in this subject. Some of this literature is devoted to the derivation of expressions for the classical force (and torque) induced by electromagnetic fields on electrically neutral polarizable continua based on an underlying discrete model of electromagnetic sources. A traditional non-relativistic approach is to take as a point of departure the classical vacuum Maxwell equations for the fields $\mathbf{e}^{U}(\mathbf{r}, t)$ and $\mathbf{b}^{U}(\mathbf{r}, t)$ in some inertial frame (here labelled $\left.U\right)$ and moving point sources 2 together with the Newtonian equations of motion in $\mathbb{R}^{3}$ for the sources. For a collection of $N$ point charges where the $\alpha$-th point has mass $m_{\alpha}$ and Newtonian velocity $\mathbf{v}_{\alpha}(t)$ at time $t$, the motion of each particle is given by a solution to the $N$ coupled ordinary differential equations:

$$
\frac{d}{d t}\left(m_{\alpha} \mathbf{v}_{\alpha}(t)\right)=\widehat{\mathcal{F}}_{\alpha}(t)+\sum_{\beta \neq \alpha}^{N} \mathcal{F}_{\alpha \beta}(t), \quad \alpha, \beta=1 \ldots N
$$

where $\mathcal{F}_{\alpha \beta}(t)$ is the inter-particle force and $\widehat{\mathcal{F}}_{\alpha}(t)$ the resultant force on the $\alpha$-th particle due to all other influences. The non-relativistic interaction of the ambient Maxwell fields $\mathbf{e}^{U}$ and $\mathbf{b}^{U}$ with any point particle with charge $q_{\alpha}$ located at $\mathbf{r}=\mathbf{r}_{\alpha}(t)$ with Newtonian velocity $\mathbf{v}_{\alpha}(t)=\dot{\mathbf{r}}_{\alpha}(t)$ is derived from the electromagnetic force $\mathcal{F}_{\alpha}^{E M}(t)$ on that particle with:

$$
\mathcal{F}_{\alpha}^{E M}(t)=q_{\alpha}\left(\mathbf{e}^{U}\left(\mathbf{r}_{\alpha}(t), t\right)+\mathbf{v}_{\alpha}(t) \times \mathbf{b}^{U}\left(\mathbf{r}_{\alpha}(t), t\right)\right)
$$

in the Gibbs vector notation. The fields themselves are in turn derived from the vacuum microscopic Maxwell equation with singular sources. In the Gibbs vector field notation these are the equations

$$
\begin{aligned}
\operatorname{curl} \underline{\mathbf{e}}^{U} & =-\frac{\partial \underline{\mathbf{b}}^{U}}{\partial t} \\
\operatorname{div} \underline{\mathbf{b}}^{U} & =0 \\
\mu_{0}{ }^{-1} \operatorname{curl} \underline{\mathbf{b}}^{U} & =\underline{\mathcal{J}}^{U}+\epsilon_{0} \frac{\partial \underline{\mathbf{e}}^{U}}{\partial t} \\
\epsilon_{0} \operatorname{div} \underline{\mathbf{e}}^{U} & =\widehat{\rho}^{U}
\end{aligned}
$$

respectively, where $\mu_{0}^{-1}=c_{0}^{2} \epsilon_{0}, c_{0}$ denotes the speed of light in vacuo, $\widehat{\rho}^{U}=$ $\sum_{\alpha} q_{\alpha} \delta^{D}\left(\mathbf{r}-\mathbf{r}_{\alpha}(t)\right)$ and $\underline{\mathcal{J}}^{U}=\sum_{\alpha} q_{\alpha} \mathbf{v}_{\alpha}(t) \delta^{D}\left(\mathbf{r}-\mathbf{r}_{\alpha}(t)\right)$ in terms of the singular Dirac distribution $\delta^{D}$. In these equations only the fundamental electromagnetic

\footnotetext{
${ }^{2}$ We assume throughout that free magnetic charge is absent in Nature.
} 
fields $\mathbf{e}^{U}$ and $\mathbf{b}^{U}$ play a role. Multi-pole magnetization and polarization sources are defined in terms of the attributes of the charged particles and their motion as limits in a multi-pole expansion. In non-relativistic models retardation effects due to the Maxwell displacement current are often ignored. It is straightforward to verify that the total linear particle momentum in the system, $\sum_{\alpha}^{N} m_{\alpha} \mathbf{v}_{\alpha}(t)$, is a constant of the motion provided $\sum_{\alpha}^{N} \widehat{\mathcal{F}}_{\alpha}(t)=0$ and $\mathcal{F}_{\alpha \beta}(t)=-\mathcal{F}_{\beta \alpha}(t)$. From these fundamental assumptions one may approach a continuum description from a number of distinct directions including a multi-pole expansion of the electromagnetic fields about some arbitrary point in space followed by a spatial smoothing procedure for the singular sources, in order to generate a balance law for smoothed out total non-relativistic linear momentum for the total interacting system. This yields an Euler continuum description from the fundamental particle-field description. While the resulting overall continuum balance equation may not be sensitive to the precise nature of the smoothing functions the interpretation of individual forces in the Eulerian balance relation may be [20, 21].

An alternative approach to a continuum description has been to assume that the point particles are electrically neutral (atoms or molecules) but are endowed with elementary electric dipole moments and/or magnetic dipoles or elementary current loops. This requires that (2) be changed to reflect this modification. Subsequent smoothing would lead to a balance law involving force terms different from the point charge model even in the static limit. This should come as no surprise since the underlying microscopic models are different.

In comparing the predictions of different continuum models one must be aware that they may differ only in their effects at the boundaries of spatially compact media. Terms that may be discarded during an integration by parts in the development of the modelling process may well contribute to boundary interactions depending on the nature of the boundary conditions.

To illustrate some of these points consider the typical modelling of a homogeneous dispersion-free electrically neutral stationary medium 22 based on a collection of point charges yielding, in some continuum limit, a time dependent volume force density on $\mathbb{R}^{3}$ given by

$\boldsymbol{F}_{t}=\frac{1}{2} \nabla\left(\mathbf{e}^{U} \cdot \mathbf{p}^{U}+\mathbf{b}^{U} \cdot \mathbf{m}^{U}\right)+\frac{1}{2} \frac{\partial}{\partial t}\left(\mathbf{p}^{U} \times \mathbf{b}^{U}\right)=F_{1 t} i+F_{2 t} \boldsymbol{j}+F_{3 t} \boldsymbol{k}$

expressed in terms of time dependent electromagnetic vector fields on $\mathbb{R}^{3}$ in a global orthonormal Cartesian frame with basis $\{\boldsymbol{i}, \boldsymbol{j}, \boldsymbol{k}\}$ and Cartesian coordinates $\left\{x^{1}, x^{2}, x^{3}\right\}$. The force vector on a finite volume $\mathcal{V}_{t}$ of the medium is then

$$
\overline{\boldsymbol{F}}_{t}=\int_{\mathcal{V}_{t}} \boldsymbol{F}_{t} d x^{1} d x^{2} d x^{3}
$$

In (7), the $\mathbf{p}^{U}$ and $\mathbf{m}^{U}$ are spatially smoothed time-dependent electric and magnetic dipole fields on $\mathbb{R}^{3}$ obtained by truncating a particle force multi-pole expansion. Prior to smoothing they can be expressed in terms of the charge, position and velocity of the electrically charged constituents of the medium relative to some arbitrary point and the bulk velocity in the medium. They are superscripted to indicate that these fields are referred to an inertial (laboratory) reference frame $U$. In principle $\mathbf{p}^{U}$ and $\mathbf{m}^{U}$ are determined from the continuum 
limit of the particle equations of motion. In practice this is difficult so one resorts to constitutive relations and assumes a form for the bulk motion of the continuum. With these closure relations the vacuum Maxwell system absorbs the smoothed polarization and magnetization sources into the phenomenological fields $\mathbf{p}^{U}, \mathbf{m}^{U}, \mathbf{d}^{U}$ and $\mathbf{h}^{U}$.

In view of the covariant language used in the rest of this paper, it is useful to write (7) in tensor form using the Killing symmetry of the Euclidean structure defined by the metric of 3-dimensional space. The reader should consult the appendixfor a self-contained formulation of the Maxwell system in terms of the frame dependent differential 1-forms $\mathbf{e}^{U}, \mathbf{b}^{U}, \mathbf{d}^{U}, \mathbf{h}^{U}, \mathbf{p}^{U}, \mathbf{m}^{U}$ on space and their definition in terms of a unit time-like vector field $U$ and the Maxwell and excitation 2-forms $F$ and $G$ respectively on 4-dimensional spacetime endowed with the Lorentzian metric tensor field 3 . In a Cartesian coordinate system on $\mathbb{R}^{3}$, the covariant and contra-variant metric tensor fields are

$$
\mathbf{g}=\sum_{i=1}^{3} d x^{i} \otimes d x^{i}, \quad \text { with } \quad \mathbf{g}^{-\mathbf{1}}=\sum_{i=1}^{3} \frac{\partial}{\partial x^{i}} \otimes \frac{\partial}{\partial x^{i}}
$$

and in these coordinates the associated translational (Killing) vector fields are $\left\{\frac{\partial}{\partial x^{i}}\right\}$ (for all time $t$ ) satisfying $\mathcal{L}_{\frac{\partial}{\partial x^{i}}} \mathbf{g}=0$. If $K$ is any of these Killing fields, then $\widetilde{K}=d x^{i}$, for some Cartesian coordinate $x^{i}, i=1,2,3$. In terms of $\kappa=\# \widetilde{K}$, with $\# 1=d x^{1} \wedge d x^{2} \wedge d x^{3}$, a Euclidean covariant representation of (7) that meshes with the conventions to be established below is given in terms of the equivalent force 3 -form for direction $K$ :

$$
\mathcal{F}_{K}=\frac{1}{2}\left[\underline{d}\left(\mathbf{e}^{U} \cdot \mathbf{p}^{U}+\mathbf{b}^{U} \cdot \mathbf{m}^{U}\right)+\# \mathcal{L}_{\partial_{t}}\left(\mathbf{p}^{U} \wedge \mathbf{b}^{U}\right)\right] \wedge \kappa
$$

where we define $\alpha \cdot \beta=g^{-1}(\alpha, \beta)=\mathbf{g}^{-\mathbf{1}}(\alpha, \beta)$ for any spatial 1 -forms $\alpha, \beta$ and $\underline{d}$ denotes the spatial exterior derivativ $\epsilon^{5}$ on $\mathbb{R}^{3}$. Then the Cartesian component of the vector force in the Cartesian direction $i$ on a volume $\mathcal{V}_{t}$ due to its surroundings and ambient fields in the medium is given by

$$
\mathcal{F}_{i}\left[\mathcal{V}_{t}\right]=\int_{\mathcal{V}_{t}} \mathcal{F}_{\partial_{i}}
$$

Note that in the static field situation the force 3 -form (76) is expressed in terms of the gradient of the interaction scalar $\frac{1}{2}\left(\mathbf{e}^{U} \cdot \mathbf{p}^{U}+\mathbf{b}^{U} \cdot \mathbf{m}^{U}\right)$. Using the macro-

\footnotetext{
3 All electromagnetic tensors in this article have dimensions constructed from the SI dimensions $[M],[L],[T],[Q]$ where $[Q]$ has the unit of the Coulomb in this system. We adopt $[g]=\left[L^{2}\right],[G]=[j]=[Q],[F]=\frac{[Q]}{\left[\epsilon_{0}\right]}$ where the permittivity of free space $\epsilon_{0}$ has the dimensions $\left[Q^{2} T^{2} M^{-1} L^{-3}\right]$. Note that the operators $d$ and $\nabla$ defined in the appendixpreserve the physical dimensions of tensor fields but with $[g]=\left[L^{2}\right]$, for $p$-forms $\alpha$ in 4 dimensions, one has $[\star \alpha]=[\alpha]\left[L^{4-2 p}\right]$.

${ }^{4}$ For any metric tensor field $\mathcal{G}$, we define the $\mathcal{G}$-dual of any 1 -form $\alpha$ to be the vector field $\widetilde{\alpha}=\mathcal{G}^{-1}(\alpha,-)$. Similarly, for any vector $V$, we define the $\mathcal{G}$-dual 1 -form $\widetilde{V}=\mathcal{G}(V,-)$.

${ }^{5}$ See appendixfor the definition of the spatial exterior derivative $\underline{d}$ in terms of the exterior derivative $d$ on spacetime, and for definitions of other spatial operators.
} 
scopic Maxwell equations

$$
\begin{aligned}
\underline{d} \mathbf{e}^{U} & =-\dot{\mathbf{B}}^{U} \\
\underline{d} \mathbf{B}^{U} & =0 \\
\underline{d} \mathbf{h}^{U} & =\mathcal{J}^{U}+\dot{\mathbf{D}}^{U} \\
\underline{d} \mathbf{D}^{U} & =\rho^{U}
\end{aligned}
$$

where $\mathbf{D}^{U}=\# \mathbf{d}^{U}, \mathbf{B}^{U}=\# \mathbf{b}^{U}, \dot{\alpha}=c_{0} \mathcal{L}_{\partial_{t}} \alpha$ for any $p$-form $\alpha$ and with the definitions

$$
\frac{\mathcal{J}_{p}^{U}}{c_{0}}=-i_{U} j_{p}=\frac{1}{c_{0}}\left(\dot{\mathbf{P}}^{U}-\underline{d \mathbf{m}^{U}}\right) \quad \text { and } \quad \rho_{p}^{U}=-\left(i_{U} \star j_{p}\right) \star \widetilde{U}=-\underline{d} \mathbf{P}^{U} \text {, }
$$

one may writ 1 , with $\mathbf{P}^{U}=\# \mp U$ and $\mathbf{E}^{U}=\# \mathbf{e}^{U}$ :

$\mathcal{F}_{K}=\frac{1}{2}\left[\rho_{p}^{U} \mathbf{e}^{U}(K)+i_{\widetilde{\mathbf{b}^{U}}} \mathcal{J}_{p}^{U} \wedge \kappa+\mathcal{L}_{\widetilde{\mathbf{b}}^{U}} \mathbf{m}^{U} \wedge \kappa+\mathcal{L}_{K} \mathbf{p}^{U} \wedge \mathbf{E}^{U}+\underline{d}\left(\mathbf{e}^{U}(K) \mathbf{P}^{U}\right)\right]$.

Although the overall charge of the medium is taken to be zero the first two terms in the equation for $\mathcal{F}_{K}$ above constitute a local Lorentz force density 2 generated by the induced polarization charge $\rho_{p}^{U}$ and current $\mathcal{J}_{p}^{U}$. The exact 3 -form in the last term will contribute to forces on the spatial boundary of the medium unless they happen to be zero as a result of boundary conditions satisfied by the fields there. The remaining terms describe local forces depending on inhomogeneities arising from the spatial rate of change of polarization and magnetization in the system.

The above derivation of the structure of a local Newtonian force density in a neutral macroscopic continuum in an electromagnetic field starts with a particular non-relativistic model in an inertial frame. We stress that in the absence of dynamical information about the polarization and magnetization the cogency of (14) demands a knowledge of supplementary constitutive relations to accommodate the response of the medium (via $\mathbf{p}^{U}$ and $\mathbf{m}^{U}$ ) to the fundamental fields $\mathbf{e}^{U}$ and $\mathbf{b}^{U}$. One could proceed to express this force density as the sum of the spatial divergence of a time dependent Maxwell second rank stress tensor on $\mathbb{R}^{3}$ and a time derivative modulo boundary term 3 . Adding the Cauchy tensor describing the medium in the absence of electromagnetic forces to such a Maxwell stress tensor would give the total Cauchy stress for the medium (in the presence of fields) that enters into the local balance law for non-relativistic linear momentum for the complete interacting system. The classical local dynamics of this system follows as a solution to such a balance law and requires implementation of interface conditions at media boundaries or interfaces where material properties of the continuum are discontinuous. The latter constraints benefit from a distributional reformulation that has been discussed more fully elsewhere [19, 23, 24].

An alternative point of departure for the modelling process is to start with a fully covariant total symmetric stress-energy-momentum tensor $T^{\text {tot }}$ for a medium interacting with classical fields on a general spacetime 5 . The fully

\footnotetext{
${ }^{1}$ See appendix for the definition of the Hodge maps \# and $\star$, and further details of this calculation.

${ }^{2}$ The factor $\frac{1}{2}$ arises since the polarization is smoothly distributed in the medium.

${ }^{3}$ There is no unique way to perform such a split.

${ }^{5}$ In the SI system the tensor $T^{\text {tot }}$ has the physical dimension of a force, i.e. $\frac{M L}{T^{2}}$.
} 
covariant local classical equations of motion of the medium are then postulated to be given by

$$
\nabla \cdot T^{t o t}=0
$$

together with equations for the fields. Here the divergence operator $\nabla \cdot$ is defined with respect to the Levi-Civita connection $\nabla$ on spacetime. The fundamental property of $T^{\text {tot }}$ is that it acts as a source of Einstein gravitation. In most classical considerations one demands $T^{t o t}(U, U)>0$ for all future pointing timelike unit vector fields $U$, reflecting the attractive nature of gravitation and $T^{t o t}(U, U)$ is then identified with local mass-energy density in the frame $U$. In a sourcefree region of spacetime containing only electromagnetic fields this condition is maintained and identifies field energy.

In a spacetime with local isometries generated by a set of Killing vector fields associated with the spacetime metric $g, T^{\text {tot }}$ can be used to generate a set of closed 3-forms on spacetime. The vanishing of the exterior derivative of each form in this set can in turn give rise to a conservation law when integrated over a regular 4-dimensional domain of spacetime provided the forms are free of singularities there. Then a class of Killing vectors that generate spatial translations can be used to construct conservation laws that reduce to the balance laws for the components of Newtonian linear momentum in some non-relativistic Newtonian limit.

Thus if $T$ is any symmetric rank 2 symmetric tensor on spacetime so $T_{a b}=$ $T_{b a}$ where

$$
T=T_{a b} e^{a} \otimes e^{b}
$$

in any cobasis of 1-forms $\left\{e^{a}\right\}$ with a dual basis of vector fields $\left\{X_{b}\right\}$ one defines the drive 3-form associated with $T$ and $K$ :

$$
\tau_{K}=-\zeta_{K} T\left(K, X_{a}\right) \star e^{a}
$$

for any vector field $K$ on spacetime where $\zeta_{K}= \pm 1$ will be defined below to conform with the physical interpretation of the different components of the drive-form. Given a frame defined by the unit timelike (future pointing) observer field 6 , one may decompose $\tau_{K}$ into a spatial 2 -form $J_{K}^{U}$ and spatial 3 -form $\rho_{K}^{U}$ relative to $U$ on spacetime:

$$
\tau_{K}=J_{K}^{U} \wedge \widetilde{U}+\rho_{K}^{U}
$$

with $i_{U} J_{K}^{U}=i_{U} \rho_{K}^{U}=0$. When $K$ is a Killing vector field $\left(\mathcal{L}_{K} g=0\right)$ it is a mathematical identity (Benn \& Tucker 1988; Benn 1982) that 7

$$
d \tau_{K}=-\zeta_{K}(\nabla \cdot T)(K) \star 1 .
$$

Hence, if $\nabla \cdot T=0$ then

$$
d \tau_{K}=0 .
$$

\footnotetext{
${ }^{6}$ The frame is inertial if $\nabla U=0$.

${ }^{7}$ More generally if $T$ is an arbitrary $(2,0)$ tensor, $T=T_{a b} e^{a} \otimes e^{b}$, of no particular symmetry, one has for any vector field $W$ the identity $\frac{1}{2}\left(\mathcal{L}_{W} g\right)\left(X_{a}, X_{b}\right) T^{\{a b\}} \star 1=-\zeta_{W} d \tau_{W}-(\nabla$. $(\operatorname{Sym} T))(\widetilde{W}) \star 1$ where $-\zeta_{W} \tau_{W}=(\operatorname{Sym} T)\left(W, X_{a}\right) \star e^{a}$ and $\operatorname{Sym} T$ is the symmetric part of $T$ with components $T_{\{a b\}}=\frac{1}{2}\left(T_{a b}+T_{b a}\right)$.
} 
In terms of $J_{K}^{U}$ the conservation equation (19) becomes

$$
d J_{K}^{U}+\mathcal{L}_{U} \tau_{K}=0 .
$$

If $K$ is a spacelike translational Killing vector field with open integral curves then

$$
J_{K}^{U} \equiv-i_{U} \tau_{K}
$$

is a linear momentum current (stress) 2-form in the frame $U$ and

$$
\rho_{K}^{U} \equiv-\left(i_{U} \star \tau_{K}\right) \star \widetilde{U}
$$

is the associated linear momentum density 3-form in the frame $U$. If $K$ is a spacelike rotational Killing vector field generating $S O(3)$ group isometries with closed integral curves, then $J_{K}^{U}$ is an angular-momentum current (torque stress) 2-form and $\rho_{K}^{U}$ is the associated angular-momentum density 3 -form in the frame $U$. If $K$ is a timelike translational Killing vector field, then $J_{K}^{U}$ is an energy current (power) 2-form and $\rho_{K}^{U}$ is the associated energy density 3form in the inertial frame field $U$. In the following, attention will be directed mainly to particular translational and rotational spacelike Killing vectors $K$ of flat spacetime and the computation of integrals of $J_{K}^{U}$ and $\rho_{K}^{U}$ for a particular contribution to $\tau_{K}$ associated with electromagnetic fields in media undergoing various states of motion observed in an inertial frame defined by $U$.

As noted above when $T^{\text {tot }}$ describes a domain of vacuum spacetime, free of matter but containing electromagnetic fields, one requires the electromagnetic field energy in any local frame $U$ to be positive, i.e. $\# \rho_{U}^{U}>0$ in terms of $\rho_{U}^{U}$. Furthermore we shall require that, for any spacelike Killing vector field $K$, $\# \rho_{K}^{U}>0$. This ensures that when $K$ generates spatial translations in Minkowski spacetime and $U$ defines an inertial frame then the time-averaged integral of $\rho_{K}^{U}$ over a finite region of space can be identified with the time-averaged component of physical linear momentum associated with a harmonic plane wave in the direction of its propagation. These conditions are ensured if

$$
\zeta_{K}=\frac{g(K, K)}{|g(K, K)|}
$$

i.e. $\zeta_{K}=1$ if $K$ is spacelike and $\zeta_{K}=-1$ if $K$ is timelike.

If the spacetime admits a foliation by hypersurfaces $t=$ constant then (19) takes the form adapted to the frame $U$ :

$$
\underline{d} J_{K}^{U}+\frac{\dot{\rho}_{K}^{U}}{c_{0}}=0 .
$$

In this spacetime framework, a basic postulate is that the history of a material continuum interacting with gravity and electromagnetic fields can be determined from (15) and the Maxwell system (47)

$$
d F=0 \quad \text { and } \quad d \star G=j,
$$

where the excitation 2-form $G$ depends on the interaction with the medium and the 3 -form electric 4-current $j$ encodes the electric charge and current source. Such an electric 4-current describes both (mobile) electric charge and effective 
(Ohmic) currents in a conducting medium. To close this system in a background gravitational field, electromagnetic constitutive relations relating $G$ and $j$ to $F$ are necessary. These relations may also depend upon properties of the medium, including its state of motion.

If one makes the arbitrary split $T^{\text {tot }}=T^{\text {matter, } E M}+T^{E M}$ then in general $\nabla \cdot T^{\text {matter }, E M}=-\nabla \cdot T^{E M} \neq 0$ so, as in the non-relativistic modelling situation, any interpretation of various terms in the decomposition of $\nabla \cdot T^{\text {tot }}$ must be understood to be with respect to a particular splitting. This is of particular relevance in situations where $\nabla \cdot T^{E M}$ contains a coupling of electromagnetic fields and mass-energy to the bulk local acceleration field of a medium.

In a fully coupled system that consistently incorporates the gravitational interactions using Einstein's gravitational field equations with a symmetric Einstein tensor, (15) becomes an identity. In descriptions with non-dynamic gravitation (15) is part of the coupled system for the remaining dynamic fields. Since the effects of gravity will be neglected in the following we assume henceforth a background Minkowski spacetime and all observers will be inertial with $\nabla U=0$.

Consider a material continuum macroscopic model in which $T^{\text {tot }}$ contains the symmetric electromagnetic stress-energy-momentum tensor

$$
T^{S M}=\frac{1}{2}\left(i_{a} F \otimes i^{a} G+i_{a} G \otimes i^{a} F+\star(F \wedge \star G) g\right) .
$$

The excitation 2-form $G$ in this expression must be specified in terms of $F$ and other properties of the medium. For a simple non-dispersive isotropic medium, one has the constitutive relation

$$
G=\epsilon_{0} \epsilon_{r} i_{V} F \wedge \widetilde{V}-\frac{\epsilon_{0}}{\mu_{r}} \star\left(i_{V} \star F \wedge \widetilde{V}\right)=\epsilon_{0}\left(\epsilon_{r}-\frac{1}{\mu_{r}}\right) i_{V} F \wedge \widetilde{V}+\frac{\epsilon_{0}}{\mu_{r}} F
$$

where $V$ is a unit, timelike 4-velocity field describing the bulk motion of the medium and $\epsilon_{r}$ and $\mu_{r}$ are relative permittivity and permeability scalars on spacetime. For a spatially inhomogeneous medium these will not be constants: $d \epsilon_{r} \neq 0, d \mu_{r} \neq 0$. Furthermore, if the medium is accelerating then $\nabla_{V} V \neq 0$. For any Killing vector field $K$, the tensor $T^{S M}$ gives rise to the Killing drive 3-form:

$$
\tau_{K}^{S M}=-\frac{\zeta_{K}}{2}\left(i_{K} G \wedge \star F-F \wedge i_{K} \star G\right),
$$

where the forms $F$ and $G$ are required to satisfy the Maxwell equations (47). For an uncharged non-conducting medium $j=0$. The stress-energy-momentum tensor (22) is that obtained by symmetrizing the one proposed by Minkowski to describe electromagnetic stresses and energy balance in a medium.

\section{Covariant Forces and Torques in Media}

In general the total stress-energy-momentum for a medium may contain singularities and discontinuities in its material properties. To facilitate the following it proves convenient to restrict to a bounded domain of spacetime containing (the smooth history of) the medium immersed in the vacuum (see figure 1). Thus we explicitly leave out of the discussion sources of stress that may arise from singularities or discontinuities in the medium history. One may regard the 


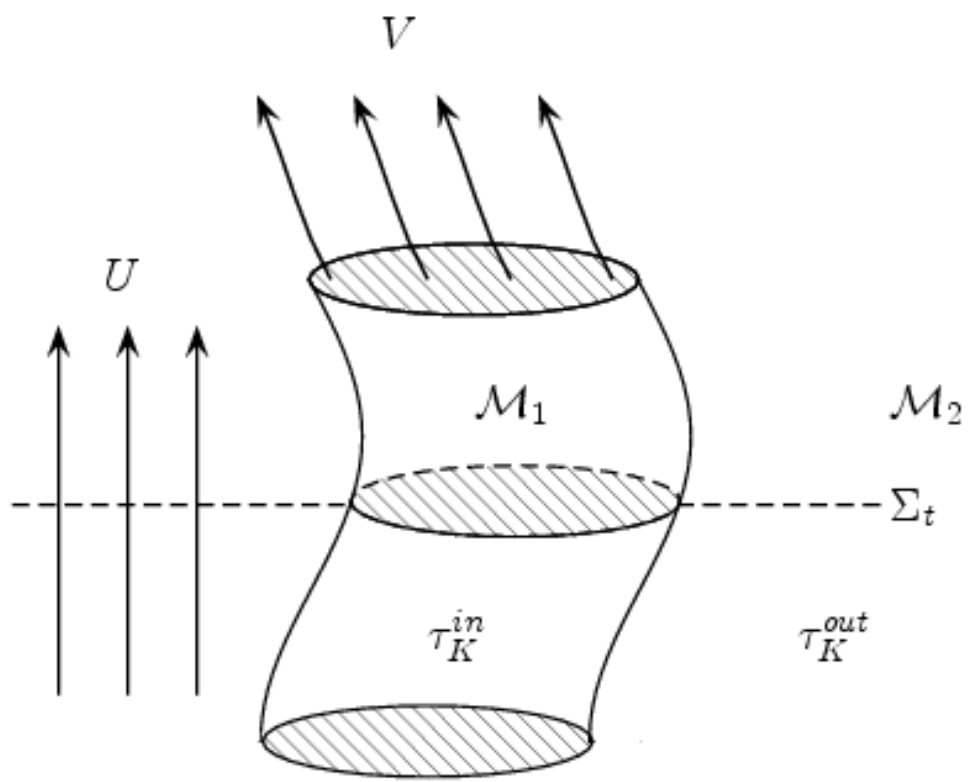

Figure 1: Immersion of a material history in spacetime

immersion as the material body and it is assumed here to have a topologically trivial structure with a total interior Killing drive form $\tau_{K}^{i n}$ with interior support including the smooth boundary of the body. The exterior of the body in spacetime is assigned a total Killing 8 drive $\tau_{K}^{\text {out }}$ with exterior support excluding the boundary of the body.

For any observer field $U$, spacetime domain $\mathcal{M}$ and total $K$-drive $\tau_{K}^{\mathcal{M}}$ on $\mathcal{M}$, one has from (17)

$$
J_{K}^{U, \mathcal{M}}=-i_{U} \tau_{K}^{\mathcal{M}} \quad \text { and } \quad \rho_{K}^{U, \mathcal{M}}=-\left(i_{U} \star \tau_{K}^{\mathcal{M}}\right) \star \widetilde{U} .
$$

If one writes

$$
\tau_{K}^{\mathcal{M}}=-\zeta_{K} c_{0}^{2} \widehat{\rho}_{m e} g(V, K) \star \widetilde{V}+\widehat{\tau}_{K}^{\mathcal{M}}
$$

to describe a medium with bulk 4-velocity $V$ on $\mathcal{M}$ and proper inertial massenergy density scalar $\widehat{\rho}_{m e}$, given the conservation law $d\left(\widehat{\rho}_{m e} \star \widetilde{V}\right)=0$, the equation of motion $d \tau_{K}^{\mathcal{M}}=0$ becomes

$$
\zeta_{K} c_{0}^{2} \widehat{\rho}_{m e} \widetilde{A}(K)=f_{K}^{\mathcal{M}}
$$

where $f_{K}^{\mathcal{M}} \equiv-\star d \widehat{\tau}_{K}^{\mathcal{M}}$ and the 4-acceleration vector field of $\mathcal{M}$ is $A \equiv \nabla_{V} V$. Contracting the $\star$ Hodge dual of this equation of motion with $U$ and integrating the resulting 3 -form over any $U$-orthogonal spacelike hypersurface $\Sigma_{t}$ that

\footnotetext{
${ }^{8}$ When the effects of gravity are neglected the interior and exterior spacetimes admit the same set of Killing vector fields.

${ }^{2}$ The first term on the right in the above equation arises from a contribution $c_{0}^{2} \widehat{\rho}_{m e} \widetilde{V} \otimes \widetilde{V}$ to the total stress-energy-momentum tensor.
} 
intersects the domain $\mathcal{M}$ yields

$$
\zeta_{K} \int_{\Sigma_{t}} \mu_{U} \widetilde{A}(K)=f_{K}^{U, \mathcal{M}}\left[\Sigma_{t}\right]
$$

where the mass-energy 3 -form $\mu_{U} \equiv-c_{0}^{2} \widehat{\rho}_{m e} \star \widetilde{U}$ and the total instantaneous integrated $K$-drive on $\Sigma_{t}$ at time $t$ in the $U$ frame is

$$
f_{K}^{U, \mathcal{M}}\left[\Sigma_{t}\right] \equiv \int_{\Sigma_{t}} i_{U} d \widehat{\tau}_{K}^{\mathcal{M}}
$$

Thus for any part $\tau_{K}^{\mathcal{M}, j}$ of the total $K$-drive $\tau_{K}^{\mathcal{M}}=\sum_{j} \tau_{K}^{\mathcal{M}, j}$ on $\mathcal{M}$ we call the 3 -form $-i_{U} d \tau_{K}^{\mathcal{M}, j}$ the $j$-th part of the instantaneous $K$-drive density 3 -form.

Using this notation consider the two regions $\mathcal{M}_{1}=$ in and $\mathcal{M}_{2}=$ out with $\tau_{K}^{i n}$ on $\mathcal{M}_{1}$ describing the interaction of a medium with electromagnetic fields according to $d \tau_{K}^{i n}=0$ and $\tau_{K}^{\text {out }}$ describing electromagnetic fields in the vacuum according to $d \tau_{K}^{\text {out }}=0$ on $\mathcal{M}_{2}$. The interface between these regions will be denoted $\Sigma_{12}$ regarded as an immersion in spacetime. In the following the difference of $J_{K}^{U, \text { in }}$ and $J_{K}^{U, \text { out }}$ across $\Sigma_{12}$ will be encountered. This discontinuity jump is defined in terms of the pull-back map $\Sigma_{12}^{\star}$ on these forms to $\Sigma_{12}$ :

$$
\Delta_{12}\left(J_{K}^{U}\right)=\Sigma_{12}^{\star}\left(J_{K}^{U, \text { in }}-J_{K}^{U, \text { out }}\right) .
$$

With a split given by $j=\{$ matter, $E M\}$ write

$$
\tau_{K}^{i n}=\tau_{K}^{i n, \text { matter }}+\tau_{K}^{i n, E M}
$$

where $\tau_{K}^{\text {in,matter }}$ describes matter without permanent polarization or magnetization in the absence of external electromagnetic fields, the equation $d \tau_{K}^{i n}=0$ yields

$$
f_{K}^{U, \text { in, matter }}\left[\Sigma_{t}\right]+f_{K}^{U, i n, E M}\left[\Sigma_{t}\right]=0 .
$$

As noted in the introduction the electromagnetic constitutive relation may imply a coupling of the electromagnetic fields in the medium to its deformation tensor and hence may contribute to its strains. For an uncharged medium, if there are no electromagnetic fields to polarize the medium, the second term on the left in (25) will be zero. In this case one may identify it with the $K$-drive on the medium due to an applied electromagnetic field. Such a $K$-drive will in general give rise to a non-zero bulk acceleration field $A=\nabla_{V} V$ on $\mathcal{M}_{1}$. In principle 9 one could maintain any prescribed state of acceleration $A_{0}$ with $V=W_{0}$ by the addition of a further drive $f_{K}^{i n, e x t}$ of some nature, provided

$$
\zeta_{K} \int_{\Sigma_{t}} \mu_{U} A_{0}(\widetilde{K})=f_{K}^{\text {in,elast }}\left[\Sigma_{t}\right]+f_{K}^{\text {in, EM }}\left[\Sigma_{t}\right]+f_{K}^{\text {in,ext }}\left[\Sigma_{t}\right]
$$

where $f_{K}^{\text {in,elast }}$ isolates possible drives in the medium due to internal non-electromagnetically induced stresses and friction with the environment. In particular if the medium is maintained in a state of uniform rotation in the absence of interaction with electromagnetic fields by mechanical torques then the

\footnotetext{
${ }^{9}$ This would be difficult in practise if electromagnetic stresses in a deformable medium led to significant strains producing a dynamic change in its shape or volume.
} 
presence of any electromagnetic induced torques can (in principle) be compensated by additional mechanical torques in order to maintain a state of uniform rotation. Using the orthogonal decompositions with respect to $U$ above and the relation $i_{U} d \tau_{K}^{i n, E M}=\underline{d} J_{K}^{i n, E M}+\mathcal{L}_{U} \rho_{K}^{i n, E M}$, such an integrated instantaneous external $K$-drive may be written:

$$
f_{K}^{U, i n, e x t}\left[\Sigma_{t}\right]=-\int_{\Sigma_{t}} i_{U} d \tau_{K}^{i n, E M}=-\int_{\Sigma_{t}} \mathcal{L}_{U} \rho_{K}^{U, i n, E M}-\int_{\partial \Sigma_{t}} J_{K}^{U, i n, E M},
$$

where Stokes' theorem has been used in the last term. The precise nature of this compensating drive will depend of course not only on the form of $\tau_{K}^{i n, E M}$ but also on the electromagnetic constitutive relation for the medium.

For unbounded media the notion of a total integrated drive may not be meaningful. In these circumstances one may be able to deduce contributions to $K$-drive pressures from the last term in (26) or by integrating discontinuities of $J_{K}^{U}$ over surfaces instead of integrating contributions to $i_{U} d \tau_{K}$ over volumes. This follows simply from the bounded medium case above by writing

$$
\int_{\partial \Sigma_{t}} J_{K}^{U, \text { in }, E M}=\int_{\partial \Sigma_{t}} J_{K}^{U, o u t, E M}+\int_{\partial \Sigma_{t}} \Delta_{12}\left(J_{K}^{U}\right),
$$

where the discontinuity $\Delta_{12}$ is across the boundary of the history in spacetime. Both integrals now require a knowledge of the pull-back to the interface of $J_{K}^{U, \text { out }}$ in the vacuum region. However one may relax the condition that the medium be bounded and assert that the instantaneous $K$-drive exerted on any finite spatial volume $\mathcal{V}_{t}$ in an infinite history having an interface $S_{t}=\partial \mathcal{V}_{t}$ with the rest of the medium and the vacuum, by drive 3 -forms that contain discontinuities over part of $S_{t}$, is given by

$$
f_{K}^{U, i n, e x t}\left[\mathcal{V}_{t}\right]=-\int_{\mathcal{V}_{t}} \mathcal{L}_{U} \rho_{K}^{U, i n, E M}-\int_{S_{t}} J_{K}^{U, \text { out }, E M}-\int_{S_{t}} \Delta_{12}\left(J_{K}^{U}\right) .
$$

In static situations the first integral on the right is zero and in some cases the second integral can be evaluated in terms of fields or their sources in the vacuum region.

\section{The Abraham and Symmetrized Minkowski Killing-Drives in Non-Accelerating Media}

As noted in the introduction there have been a number of different proposals for the electromagnetic stress-energy-momentum tensor. In the tables in Appendix A, the properties of a number of these [26, 27], are displayed using the notation introduced above. For a recent derivation of these tensors from a variational approach see [28, 29]. They permit a ready evaluation of the $K$-drives on media with bulk 4 -velocity field $V$ and specified constitutive properties. For convenience, separate entries for the Abraham tensor have been provided for media at rest relative to a frame $U$ (i.e. $V=U$ ). To calculate forces and torques it is only necessary to recognize that they are defined by particular spacelike Killing vectors $K$. So in an inertial frame $U$ with $\widetilde{U}(K)=0$ many entries will 
simplify. Separate entries are also given for situations when $K$ is timelike and equal to $U$ since this facilitates computation of energies and powers. In the calculation of the divergence of the Abraham stress-energy-momentum we have made explicit those terms that depend on the bulk acceleration field $A \equiv \nabla_{V} V$ of the medium. Such terms are of course absent for media at rest or moving with arbitrary linear velocity in an inertial frame $(\nabla U=0)$. It is only in special circumstances that there are simple relations between the Minkowski and Abraham tensors. These relations arise mainly for simple media at rest in an inertial frame. Thus if

$$
\mathbf{d}^{U}=\epsilon_{0} \epsilon_{r} \mathbf{e}^{U}, \quad \mathbf{b}^{U}=\mu_{0} \mu_{r} \mathbf{h}^{U}
$$

in a stationary homogeneous medium (with constant scalars $\epsilon_{r}$ and $\mu_{r}$ ), then for all $K$, the $J_{K}^{U}$ are identical for $T^{S M}, T^{M}$ and $T^{A B}$ (see table in Appendix A). However the corresponding $\rho_{K}^{U}$ are all different. In problems with time-harmonic electromagnetic fields the $\mathcal{L}_{U} \rho_{K}^{U}$ have zero averages over time, implying that the corresponding time-averaged $K$-drives $\left\langle f_{K}^{U}\right\rangle$ are the same.

In a simple non-accelerating $(A=0)$ medium that may be inhomogeneous there is a simple relation between $\tau_{K}^{A}$ and $\tau_{K}^{S M}$ (see table in Appendix A) for arbitrary time-dependent fields. Given a spacelike translational Killing vector $K$ one sees from the table that (since $\widetilde{U}(K)=0$ )

$$
\tau_{K}^{A}=\tau_{K}^{S M}-\frac{1}{2} s^{U}(K) \star \widetilde{U},
$$

where the 1-form

$$
\boldsymbol{s}^{U}=\star\left(\frac{1}{c_{0}} \mathbf{e}^{U} \wedge \mathbf{h}^{U} \wedge \widetilde{U}-c_{0} \mathbf{d}^{U} \wedge \mathbf{b}^{U} \wedge \widetilde{U}\right) .
$$

Using the simple constitutive relations (28) this 1-form can be expressed in terms of the spatial fields $\mathbf{e}^{U}, \mathbf{h}^{U}$ and the form $\widetilde{U}$

$$
s^{U}=\frac{1}{c_{0}}\left(1-\mathcal{N}^{2}\right) \star\left(\mathbf{e}^{U} \wedge \mathbf{h}^{U} \wedge \widetilde{U}\right),
$$

where $\mathcal{N} \equiv \sqrt{\epsilon_{r} \mu_{r}}$ is the refractive index of the medium. Thus

$s^{U}(K) \star \widetilde{U}=-\frac{1}{c_{0}}\left(1-\mathcal{N}^{2}\right) i_{U}\left(\mathbf{e}^{U} \wedge \mathbf{h}^{U} \wedge \widetilde{U} \wedge \widetilde{K}\right)=\frac{1}{c_{0}}\left(1-\mathcal{N}^{2}\right)\left(\mathbf{e}^{U} \wedge \mathbf{h}^{U} \wedge \widetilde{K}\right)$

and so

$$
\tau_{K}^{A}=\tau_{K}^{S M}+\frac{1}{2 c_{0}}\left(\mathcal{N}^{2}-1\right)\left(\mathbf{e}^{U} \wedge \mathbf{h}^{U} \wedge \widetilde{K}\right) .
$$

By contracting the exterior derivative of this expression with $U=\frac{1}{c_{0}} \partial_{t}$ (defining a non-accelerating reference frame) one deduces a relation between the 3 -form force density $\mathcal{F}_{K}^{U, A}=i_{U} d \tau_{K}^{A}$ associated with the Abraham drive 3-form, and $\mathcal{F}_{K}^{U, S M}=i_{U} d \tau_{K}^{S M}$ associated with the symmetrized Minkowski drive 3-form:

$$
\mathcal{F}_{K}^{U, A}=\mathcal{F}_{K}^{U, S M}+\frac{1}{2 c_{0}^{2}} \mathcal{L}_{\partial_{t}}\left(\left(\mathcal{N}^{2}-1\right) \mathbf{e}^{U} \wedge \mathbf{h}^{U}\right) \wedge \widetilde{K},
$$


using

$$
d \phi=\underline{d} \phi-\widetilde{U} \wedge \mathcal{L}_{U} \phi=\underline{d} \phi+d t \wedge \mathcal{L}_{\partial_{t}} \phi,
$$

and noting that $\left(\mathcal{N}^{2}-1\right)\left(\mathbf{e}^{U} \wedge \mathbf{h}^{U} \wedge \widetilde{K}\right)$ is a spatial 3-form 1 . The second term on the right in (31) has been termed the Abraham force. In static field situations it is zero and the two force densities are equal. This is also the situation in the presence of time-periodic fields if one takes the time-average of this equation over a time period. For smooth pulsed fields the second term will in general yield a finite contribution to a total force when (31) is integrated over a finite time interval.

Returning to the constitutive case for an arbitrarily moving medium () one can use the results in the previous section to facilitate a comparison between the Newtonian force density (14) and that based on the choice with $\tau_{K}^{\mathcal{M}, E M}=\tau_{K}^{S M}$. Thus one must calculate $i_{U} d \tau_{K}^{S M}$ for a Killing field $K$ that generates spatial translations and express the result in terms of the polarization and magnetization 1-forms $\mathbf{p}^{U}$ and $\mathbf{m}^{U}$ respectively. A local constitutive relation between $G$ and $F$ will induce a local constitutive relation between $\mathbf{p}^{U}$ and $\mathbf{m}^{U}$ and the electromagnetic fields $\mathbf{e}^{U}$ and $\mathbf{b}^{U}$ and the permittivity and permeability of the medium. Since $d F=0$,

$$
2 d \tau_{K}^{S M}=-\left(d i_{K} G \wedge \star F-i_{K} G \wedge d \star F-F \wedge d i_{K} \star G\right) .
$$

Writing $G=\epsilon_{0} F+\Pi$, one has

$$
\begin{aligned}
& d i_{K} G \wedge \star F=\mathcal{L}_{K} G \wedge \star F-i_{K} d G \wedge \star F=F \wedge \star \mathcal{L}_{K} G-i_{K} d\left(\epsilon_{0} F+\Pi\right) \wedge \star F \\
& \quad=F \wedge \mathcal{L}_{K} \star G-i_{K} d \Pi \wedge \star F=F \wedge i_{K} d \star G+F \wedge d i_{K} \star G-F \wedge \star i_{K} d \Pi \\
& \quad=F \wedge i_{K} j+F \wedge d i_{K} \star G-F \wedge \star i_{K} d \Pi .
\end{aligned}
$$

Hence

$$
2 d \tau_{K}^{S M}=-F \wedge i_{K} j+F \wedge \star i_{K} d \Pi+i_{K} G \wedge d \star F
$$

Since

$$
d \star G=\epsilon_{0} d \star F+d \star \Pi=j, \quad \text { or } \quad d \star F=\frac{1}{\epsilon_{0}}(j-d \star \Pi),
$$

and

$$
\begin{aligned}
& i_{K} G \wedge d \star F=-G \wedge i_{K} d \star F=-\frac{1}{\epsilon_{0}} G \wedge i_{K}(j-d \star \Pi) \\
& =-\frac{1}{\epsilon_{0}} G \wedge i_{K} j+\frac{1}{\epsilon_{0}} G \wedge i_{K} d \star \Pi=-F \wedge i_{K} j-\frac{\Pi}{\epsilon_{0}} \wedge i_{K} j+G \wedge i_{K} d \star \frac{\Pi}{\epsilon_{0}},
\end{aligned}
$$

one has

$$
2 d \tau_{K}^{S M}=-\left(2 F+\frac{\Pi}{\epsilon_{0}}\right) \wedge i_{K} j+F \wedge \star i_{K} d \Pi+G \wedge i_{K} d \star \frac{\Pi}{\epsilon_{0}} .
$$

\footnotetext{
${ }^{1}$ If one replaces $U$ by $V$ with $\nabla_{V} V \neq 0$ in (29) one is dealing with accelerating media and the Abraham drive $\mathcal{F}_{K}^{U, A}$ then acquires additional terms in (31). Such additional terms are often overlooked when considering the effects of the so called Abraham force (or torque). This may lead to inconsistencies in processes involving accelerating media.
} 
This can be decomposed in terms of $\mathbf{e}^{U}, \mathbf{b}^{U}, \mathbf{p}^{U}, \mathbf{m}^{U}$, and real and induced polarization and magnetization sources yielding 2

$$
\begin{aligned}
& -2 d \tau_{K}^{S M}=\left(\rho_{p}^{U} \mathbf{e}^{U}(K)+i_{\widetilde{\mathbf{b}^{U}}} \mathcal{J}_{p}^{U} \wedge \kappa+\frac{1}{\epsilon_{0}}\left(\rho^{U}+\rho_{p}^{U}\right) \mathbf{p}^{U}(K)\right) \wedge \widetilde{U} \\
& +\left(\mu_{0}{\underset{\mathbf{m}}{\mathbf{m}^{U}}}\left(\mathcal{J}^{U}+\mathcal{J}_{p}^{U}\right) \wedge \kappa+2 \rho^{U} \mathbf{e}^{U}(K)+2 i_{\widetilde{\mathbf{b}^{U}}} \mathcal{J}^{U} \wedge \kappa+\frac{1}{c_{0}} i_{\widetilde{\mathbf{e}^{U}}} \mathcal{J}_{m}^{U} \wedge \kappa\right. \\
& \left.-c_{0} \rho_{m}^{U} \mathbf{b}^{U}(K)\right) \wedge \widetilde{U}-2 c_{0} \mathbf{B}^{U} \wedge i_{K} \rho^{U}-\frac{1}{\epsilon_{0} c_{0}} \mathbf{M}^{U} \wedge i_{K} \rho^{U} \\
& -i_{K} \rho_{m}^{U} \wedge \mathbf{E}^{U}+c_{0} i_{K} \mathbf{B}^{U} \wedge \rho_{p}^{U}+\frac{1}{\epsilon_{0} c_{0}} i_{K} \mathbf{M}^{U} \wedge \rho_{p}^{U},
\end{aligned}
$$

where $\mathbf{M}^{U}=\# M m U$. Contracting with $U$ yields

$$
\begin{aligned}
\mathcal{F}_{K}^{U, S M}=-i_{U} d \tau_{K}^{S M}= & \rho^{U} \mathbf{e}^{U}(K)+i_{\widetilde{\mathbf{b}^{U}}} \mathcal{J}^{U} \wedge \kappa+\frac{1}{2} \rho_{p}^{U} \mathbf{e}^{U}(K) \\
& +\frac{1}{2} i_{\widetilde{\mathbf{b}^{U}}} \mathcal{J}_{p}^{U} \wedge \kappa+\frac{1}{2 c_{0}} i_{\widetilde{\mathbf{e}^{U}}} \mathcal{J}_{m}^{U} \wedge \kappa-\frac{1}{2} c_{0} \rho_{m}^{U} \mathbf{b}^{U}(K) \\
& +\frac{1}{2 \epsilon_{0}}\left(\rho^{U}+\rho_{p}^{U}\right) \mathbf{p}^{U}(K)+\frac{1}{2} \mu_{0} i_{\widetilde{\mathbf{m}^{U}}}\left(\mathcal{J}^{U}+\mathcal{J}_{p}^{U}\right) \wedge \kappa .
\end{aligned}
$$

The first two terms on the right constitute the Lorentz force density due to any real charge $\rho^{U}$ and electric current $\mathcal{J}^{U}$ in the medium. The third and fourth terms constitute the Lorentz force density due to induced polarization charge $\rho_{p}^{U}$ and electric current $\mathcal{J}_{p}^{U}$. These are precisely the force densities that arise in the non-relativistic electrically neutral smoothed model (14) above based on the motion of point charges. The fifth and sixth terms constitute the Lorentz force density due to magnetization charge $\rho_{m}^{U}$ and magnetization current $\mathcal{J}_{m}^{U}$. These do not arise in the Newtonian model (14) above since the point charges in that model were not endowed with intrinsic magnetic moments.

\section{Conclusions}

The general theory of drive-forms has been developed starting from the vanishing divergence of a total stress-energy-momentum in an arbitrary spacetime using the language of exterior systems. It has been shown that a decomposition of a drive 3-form, relative to a unit timelike vector field, on a spacetime with sufficient Killing vectors yields forms that can be associated with force and torque densities in continuous media. For material subject to the electromagnetic constitutive relations proposed by Minkowski it has also been shown how the computation of the electromagnetic force density on a non-accelerating polarizable perfectly insulating medium in an electromagnetic field can be effectively carried out in terms of a particular split of the total stress-energy-momentum into parts describing its inertial and electromagnetic properties. In paper II this theory is applied to homogeneous and inhomogeneous dielectric media where it is argued that a means of discriminating between a split into the electromagnetic stressenergy-momentum tensors proposed by Abraham and Minkowski (and possibly others) can be explored experimentally using rotating media.

\section{Acknowledgements}

The authors are grateful to the Cockcroft Institute, the Alpha-X project, STFC and EPSRC (EP/E001831/1) for financial support for this research.

\footnotetext{
${ }^{2}$ See appendixfor further details of this calculation.
} 


\section{A Summary of Electromagnetic Stress-Energy-Momentum Tensors}

\begin{tabular}{|c|c|c|}
\hline & MINKOWSKI & SYMMETRIZED MINKOWSKI \\
\hline$T^{E M}$ & $i_{a} F \otimes i^{a} G+\frac{1}{2} \star(F \wedge \star G) g$ & $T^{S M}=\frac{1}{2}\left(i_{a} F \otimes i^{a} G+i_{a} G \otimes i^{a} F+\star(F \wedge \star G) g\right)$ \\
\hline$\tau_{K}^{E M}$ & $-\frac{\zeta_{K}}{2}\left(i_{K} F \wedge \star G-F \wedge i_{K} \star G\right)$ & $-\frac{\zeta_{K}}{2}\left(i_{K} G \wedge \star F-F \wedge i_{K} \star G\right)$ \\
\hline$\underset{T^{E M}}{\nabla \cdot}$ & $\frac{1}{2} d \star(F \wedge \star G)-\left(\nabla_{X_{a}} G\right)\left(\widetilde{i^{a} F}\right)-G(\widetilde{\nabla \cdot F})$ & $\frac{1}{2}\left(d \star(F \wedge \star G)-\left(\nabla_{X_{a}} G\right)\left(\widetilde{i^{a} F}\right)-\left(\nabla_{X_{a}} F\right)\left(\widetilde{i^{a} G}\right)-G(\widetilde{\nabla \cdot F})-F(\widetilde{\nabla \cdot G})\right)$ \\
\hline$J_{K}^{U}$ & $\begin{array}{l}-\zeta_{K}\left[\mathbf{e}^{U}(K) \mathbf{D}^{U}+\mathbf{h}^{U}(K) \mathbf{B}^{U}-\frac{1}{c_{0}} \widetilde{U}(K) \mathbf{e}^{U} \wedge \mathbf{h}^{U}\right. \\
\left.-\frac{1}{2}\left(\mathbf{e}^{U} \cdot \mathbf{d}^{U}+\mathbf{b}^{U} \cdot \mathbf{h}^{U}\right) \kappa\right]\end{array}$ & $\begin{array}{l}-\frac{\zeta_{K}}{2}\left[\mathbf{e}^{U}(K) \mathbf{D}^{U}+\mathbf{d}^{U}(K) \mathbf{E}^{U}+\mathbf{h}^{U}(K) \mathbf{B}^{U}+\mathbf{b}^{U}(K) \mathbf{H}^{U}\right. \\
\left.-\left(\mathbf{e}^{U} \cdot \mathbf{d}^{U}+\mathbf{b}^{U} \cdot \mathbf{h}^{U}\right) \kappa-\widetilde{U}(K)\left(\frac{1}{c_{0}} \mathbf{e}^{U} \wedge \mathbf{h}^{U}+c_{0} \mathbf{d}^{U} \wedge \mathbf{b}^{U}\right)\right]\end{array}$ \\
\hline$\rho_{K}^{U}$ & $\begin{array}{l}\zeta_{K}\left[c_{0} \mathbf{d}^{U} \wedge \mathbf{b}^{U} \wedge \widetilde{K}^{\perp}\right. \\
\left.+\frac{1}{2} \widetilde{U}(K)\left(\mathbf{e}^{U} \cdot \mathbf{d}^{U}+\mathbf{b}^{U} \cdot \mathbf{h}^{U}\right) \# 1\right]\end{array}$ & $\frac{\zeta_{K}}{2}\left[\left(\frac{1}{c_{0}} \mathbf{e}^{U} \wedge \mathbf{h}^{U}+c_{0} \mathbf{d}^{U} \wedge \mathbf{b}^{U}\right) \wedge \widetilde{K}^{\perp}+\widetilde{U}(K)\left(\mathbf{e}^{U} \cdot \mathbf{d}^{U}+\mathbf{b}^{U} \cdot \mathbf{h}^{U}\right) \# 1\right]$ \\
\hline$J_{U}^{U}$ & $\frac{1}{c_{0}} \mathbf{e}^{U} \wedge \mathbf{h}^{U}$ & $\frac{1}{2}\left(\frac{1}{c_{0}} \mathbf{e}^{U} \wedge \mathbf{h}^{U}+c_{0} \mathbf{d}^{U} \wedge \mathbf{b}^{U}\right)$ \\
\hline$\rho_{U}^{U}$ & $\frac{1}{2}\left(\mathbf{e}^{U} \cdot \mathbf{d}^{U}+\mathbf{b}^{U} \cdot \mathbf{h}^{U}\right) \# 1$ & $\frac{1}{2}\left(\mathbf{e}^{U} \cdot \mathbf{d}^{U}+\mathbf{b}^{U} \cdot \mathbf{h}^{U}\right) \# 1$ \\
\hline
\end{tabular}




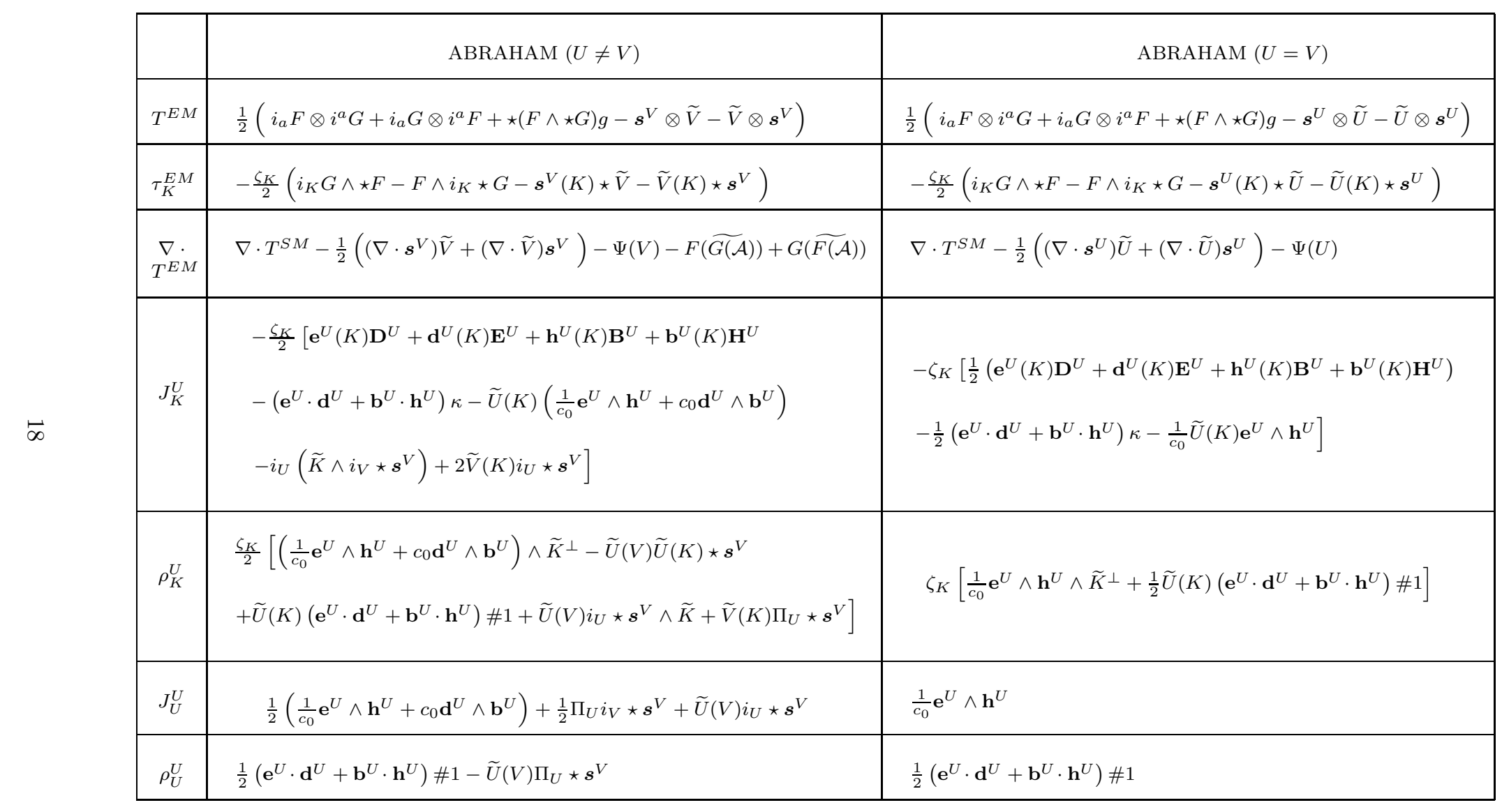


In these tables we have introduced the 1-form:

$$
\begin{aligned}
s^{V} & =\star\left(i_{V} F \wedge i_{V} \star G \wedge \widetilde{V}-i_{V} G \wedge i_{V} \star F \wedge \widetilde{V}\right) \\
& =\star\left(\frac{1}{c_{0}} \mathbf{e}^{V} \wedge \mathbf{h}^{V} \wedge \widetilde{V}-c_{0} \mathbf{d}^{V} \wedge \mathbf{b}^{V} \wedge \widetilde{V}\right)
\end{aligned}
$$

where the spatial fields $\mathbf{e}^{V}, \mathbf{b}^{V}, \mathbf{d}^{V}, \mathbf{h}^{V}$ are defined by the orthogonal splits of $F$ and $G$ relative to the 4 -velocity field $V$ of the medium. Furthermore, we have defined $\mathbf{H}^{U}=\# \mathbf{h}^{U}$. The vector fields $U$ and $V$ on spacetime are timelike, unit, normalized with a metric $g$ of signature $(-1,+1,+1,+1)$ so that $\widetilde{U}(U)=$ $\widetilde{V}(V)=-1$, and describe the state of motion of the observer and medium respectively. It is also convenient to introduce, in terms of the vector field $U$ and any vector field $Y$ on spacetime the projection $Y^{\perp}=Y+\widetilde{U}(Y) U$, which induces the projection operator on $p$-forms $\Pi_{U}=\mathbb{I}+\widetilde{U} \wedge i_{U}$. The spatial Hodge map \# with respect to $U$ is defined by $\star 1=\widetilde{U} \wedge \# 1$, and maps spatial $p$-forms to spatial $(3-p)$-forms. For any Killing vector field $K$ on spacetime (i.e. $\mathcal{L}_{K} g=0$ ) we denote the 2 -form $\# \widetilde{K}$ by $\kappa$. If $K$ is a Killing vector then so is $\xi_{0} K$ where $\xi_{0}$ is any constant. Hence the physical dimensions of quantities that depend on the Killing vector field $\xi_{0} K$ will depend on the physical dimensions of $\xi_{0}$.

In the table we have isolated those terms in the divergence that depend explicitly on the bulk medium acceleration $A \equiv \nabla_{V} V$. Among the remaining terms there is a set that can be expressed in terms of a $(2,0)$-tensor field $\Psi$ on spacetime defined, for any vector fields $X, Y$, by $\Psi(X, Y)=(\Psi(X))(Y)$ where

$$
\begin{aligned}
\Psi(X) \equiv & \left(\nabla_{X} F\right)\left(\widetilde{\left(i_{X} G\right.}\right)+F\left(\widetilde{i_{X} \nabla_{X} G}\right)-\left(\nabla_{X} G\right)\left(\widetilde{i_{X} F}\right)-G\left(\widetilde{i_{X} \nabla_{X} F}\right)-\frac{1}{2}\left(\mathcal{L}_{X} F\right)\left(\widetilde{i_{X} G}\right) \\
& -\frac{1}{2} F\left(\left(\mathcal{L}_{X} g^{-1}\right)\left(i_{X} G\right)\right)-\frac{1}{2} F\left(\widetilde{i_{X} \mathcal{L}_{X} G}\right)+\frac{1}{2}\left(\mathcal{L}_{X} G\right)\left(\widetilde{i_{X} F}\right)+\frac{1}{2} G\left(\left(\mathcal{L}_{X} g^{-1}\right)\left(i_{X} F\right)\right) \\
& +\frac{1}{2} G\left(\widetilde{i_{X} \mathcal{L}_{X} F}\right)+\frac{1}{2}\left(\mathcal{L}_{X} g\right)\left(\widetilde{F\left(\widetilde{i_{X} G}\right)}\right)-\frac{1}{2}\left(\mathcal{L}_{X} g\right)\left(G\left(\widetilde{i_{X} F}\right)\right)
\end{aligned}
$$

with $F(Y) \equiv i_{Y} F$ and $G(Y) \equiv i_{Y} G$. If $\left\{X_{a}\right\}$ denotes an orthonormal frame with dual cobasis $\left\{e^{b}\right\}$ one has $g^{-1}=\eta^{a b} X_{a} \otimes X_{b}, \quad g=\eta_{a b} e^{a} \otimes e^{b}$ where $\eta^{a b}$ and $\eta_{a b}$ are both diagonal matrices with $\eta^{00}=\eta_{00}=-1$ and $\eta_{i j}=\eta^{i j}=\delta_{i j}$ for $i, j=1,2,3$. The contraction operator $i_{X_{a}}$ is abbreviated $i_{a}$ and $i^{a} \equiv \eta^{a b} i_{b}$. The spacetime Hodge map is denoted $\star$ and the canonical 4 -form on spacetime is $\star 1=e^{0} \wedge e^{1} \wedge e^{2} \wedge e^{3}$ in an orthonormal basis and is $\sqrt{|\operatorname{det} g|} d^{4} x$ in any coordinate system $\left\{x^{a}\right\}$.

\section{B Notation}

The natural mathematical language to discuss the differential properties of tensor fields on spacetime and their relation to integrals over material domains is in terms of differential forms and their associated exterior calculus [18. In this section a brief summary is given of the relevant notation used in subsequent sections. A key concept throughout involves the role of the spacetime metric tensor field and possible isometries that it may possess. A spacetime metric tensor field $g$ is a symmetric bilinear form on spacetime that can always be represented in a local cobasis of differential 1-forms $\left\{e^{a}\right\}$ as

$$
g=-e^{0} \otimes e^{0}+e^{1} \otimes e^{1}+e^{2} \otimes e^{2}+e^{3} \otimes e^{3} .
$$


If $\left\{X_{b}\right\}$ is the dual local basis of vector fields on spacetime defined so that $e^{a}\left(X_{b}\right)=\delta_{b}^{a}(a, b,=0,1,2,3)$ one has the induced contravariant metric

$$
g^{-1}=-X_{0} \otimes X_{0}+X_{1} \otimes X_{1}+X_{2} \otimes X_{2}+X_{3} \otimes X_{3} .
$$

If $\alpha$ is any given 1 -form, one has an associated vector field $\widetilde{\alpha}$ defined so that $\beta(\widetilde{\alpha})=g^{-1}(\alpha, \beta)$ for all 1 -forms $\beta$. Since $g^{-1}$ is symmetric this will be abbreviated $\widetilde{\alpha}=g^{-1}(\alpha)$. In a similar way if $X$ is any given vector field one has an associated 1-form $\widetilde{X}=g(X)$.

In the $g$-orthonormal basis $\left\{e^{a}\right\}$ one has a canonical local 4 -form denoted $\star 1$ and defined to be $e^{0} \wedge e^{1} \wedge e^{2} \wedge e^{3}$. The Hodge map $\star$ induced by $\star 1$ maps $p$-forms to $(4-p)$-forms on spacetime (Benn \& Tucker 1988; see also Appendix A of paper I). The metric also uniquely defines the covariant derivative $\nabla_{X}$ with respect to any vector field $X$. This has the property $\nabla_{X} g=0$ and $\nabla_{X} Y-\nabla_{Y} X=[X, Y]$ for all vector fields $X, Y$. In this expression $[X, Y]$ denotes the commutator bracket. While $\nabla_{X}$ has a type-preserving action on any tensor field the exterior derivative $d$ is defined to act only on antisymmetric tensor fields (differential forms) and has the property $d \circ d=0$. Contraction of any $p$-form $\beta$ with $X$ is denoted $i_{X} \beta$. The interior operator $i_{X}$ is a graded derivation defined so that

$$
i_{X}(\alpha \wedge \beta)=\left(i_{X} \alpha\right) \wedge \beta+(-1)^{p} \alpha \wedge i_{X} \beta,
$$

for any $p$-form $\alpha$ and $q$-form $\beta$. If $p=1$, one defines $i_{X} \alpha=\alpha(X)$, and if $p=0$, $i_{X} \alpha=0$, for all vector fields $X$. One has the useful relations

$$
\begin{aligned}
\star \star \Phi & =(-1)^{p+1} \Phi \\
i_{X} \star \Phi & =\star(\Phi \wedge \widetilde{X}) \\
\mathcal{L}_{X} \Phi & =i_{X} d \Phi+d i_{X} \Phi,
\end{aligned}
$$

for any $p$-form $\Phi$ on spacetime where $\mathcal{L}_{X}$ denotes Lie differentiation with respect to $X$.

The spacetime divergence operator $\nabla \cdot$ takes a simple form if one uses the $g$-orthonormal basis above. Acting on a symmetric covariant tensor $T$ it defines by contraction on the first argument the 1-form

$$
\nabla \cdot T=\sum_{a=0}^{3}\left(\nabla_{X_{a}} T\right)\left(X^{a},-\right)
$$

where $\left\{X^{a}\right\}=\left\{-X_{0}, X_{1}, X_{2}, X_{3}\right\}$. For a symmetric tensor $T$ it is sufficient to write the right hand side as $\left(\nabla_{X_{a}} T\right)\left(X^{a}\right)$. A local spacetime isometry with respect to $g$ is a local diffeomorphism that preserves this metric. A vector field $K$ that generates such a diffeomorphism is called a Killing vector field and it satisfies $\mathcal{L}_{K} g=0$ in terms of the operation of Lie differentiation with respect to $K$. The operators $\star, d, i_{X}, \nabla_{X}, \mathcal{L}_{X}$ offer a powerful computational tool-kit when working with differential forms.

For any smooth $p$-form $\Phi$ in a bounded regular region $\mathcal{M}$ of a manifold one can express the integral of $d \Phi$ over $\mathcal{M}$ in terms of the integral of $\Phi$ over the boundary $\partial \mathcal{M}$ of $\mathcal{M}$ :

$$
\int_{\mathcal{M}} d \Phi=\int_{\partial \mathcal{M}} \Phi
$$


This is a statement of Stokes' theorem for $p$-forms.

The Gibbs calculus of vector fields in 3-dimensional Euclidean space is readily exposed by correspondences induced by the exterior operations above. A space of 3-dimensions may be considered as a particular hypersurface in spacetime. If the metric above induces the metric

$$
\mathbf{g}=e^{1} \otimes e^{1}+e^{2} \otimes e^{2}+e^{3} \otimes e^{3}
$$

on this hypersurface it is Euclidean and one may introduce the Euclidean canonical form \#1 $=e^{1} \wedge e^{2} \wedge e^{3}$ by restriction. Since spacetime is assumed timeoriented one may employ a future-pointing timelike unit vector field $U$ on spacetime (with $g(U, U)=-1$ and $\widetilde{U}=e^{0}$ ) to fix a coherent orientation by relating $\# 1$ to $\star 1$ by

$$
\star 1=\widetilde{U} \wedge \# 1 .
$$

If the hypersurface is given as $t=$ constant for some time coordinate $t$, an inertial frame exists in Minkowski spacetime with $U=\frac{1}{c_{0}} \frac{\partial}{\partial t}=-X_{0}$ such that $\nabla U=0$. Throughout this article the constant $c_{0}$ denotes the speed of light in the vacuum. Any $p$-form $\alpha$ on spacetime is termed spatial with respect to such a $U$ if $i_{U} \alpha=0$. An over-dot will denote (Lie) differentiation with respect to the coordinate $t$ so

$$
\dot{\alpha} \equiv c_{0} \mathcal{L}_{U} \alpha=\mathcal{L}_{\partial_{t}} \alpha .
$$

On a Euclidean hypersurface in spacetime, exterior differentiation of spatial $p$-forms $\phi$ is denoted $\underline{d} \phi$ such that

$$
d \phi=\underline{d} \phi-\widetilde{U} \wedge \mathcal{L}_{U} \phi=\underline{d} \phi+d t \wedge \mathcal{L}_{\partial_{t}} \phi .
$$

One has the following relations

$$
\begin{aligned}
\# 1 & =-i_{U} \star 1=-\star \widetilde{U} \\
\# \# \phi & =\phi
\end{aligned}
$$

for all spatial $p$-forms $\phi$. Furthermore if $\mathbf{v}, \mathbf{w}$ denote Euclidean vector fields in the Gibbs notation corresponding to the vector fields $\widetilde{v}, \widetilde{w}$ for some spatial 1-forms $v, w$ then

$\begin{array}{lll}\operatorname{div} \mathbf{v} & \text { corresponds to } & \widetilde{\# \underline{\#} \boldsymbol{v} ;} \\ \operatorname{curl} \mathbf{v} & \text { corresponds to } & \widetilde{\# \underline{d} v ;} \\ \mathbf{v} \times \mathbf{w} & \text { corresponds to } & \widetilde{\#(v \wedge w) ;} \\ \operatorname{grad} \psi & \text { corresponds to } & \widetilde{d} \psi\end{array}$

for any scalar $\psi$ field on spacetime. 


\section{Electromagnetic Fields on Spacetime}

We suppose that a polarizable material continuum is given in terms of a set of piecewise smooth material properties that determine its interaction with classical gravitational and electromagnetic fields. The classical macroscopic Maxwell system for the electromagnetic 2 -form $F$ on spacetime can be written as (Tucker \& Walton 2009):

$$
d F=0 \quad \text { and } \quad d \star G=j,
$$

where the excitation 2 -form $G$ depends on the interaction with the medium and the 3 -form electric 4-current $j$ encodes the electric charge and current source 10 . Such an electric 4-current describes both (mobile) electric charge and effective (Ohmic) currents in a conducting medium. To close this system in a background gravitational field, electromagnetic constitutive relations relating $G$ and $j$ to $F$ are necessary.

The history of a particular observer field in spacetime is associated with an arbitrary unit future-pointing timelike 4-velocity vector field $U$. The field $U$ may be used to describe an observer frame on spacetime and its integral curves model idealized observers. An orthogonal decomposition of $F$ with respect to any observer field $U$ gives rise to a pair of spatial 1-forms on spacetime. The 1-form spatial electric field $\mathbf{e}^{U}$ and 1-form spatial magnetic induction field $\mathbf{b}^{U}$ associated with $F$ are defined with respect to an observer field $U$ by

$$
\mathbf{e}^{U}=i_{U} F \quad \text { and } \quad c_{0} \mathbf{b}^{U}=i_{U} \star F .
$$

Since $g(U, U)=-1$ and $i_{U} \mathbf{e}^{U}=i_{U} \mathbf{b}^{U}=0$ :

$$
F=\mathbf{e}^{U} \wedge \widetilde{U}-\star\left(c_{0} \mathbf{b}^{U} \wedge \tilde{U}\right)
$$

Likewise the 1-form spatial displacement field $\mathbf{d}^{U}$ and the 1-form spatial magnetic field $\mathbf{h}^{U}$ associated with $G$ are defined with respect to $U$ by

$$
\mathbf{d}^{U}=i_{U} G \quad \text { and } \quad \frac{\mathbf{h}^{U}}{c_{0}}=i_{U} \star G,
$$

so

$$
G=\mathbf{d}^{U} \wedge \widetilde{U}-\star\left(\frac{\mathbf{h}^{U}}{c_{0}} \wedge \widetilde{U}\right),
$$

with $i_{U} \mathbf{d}^{U}=i_{U} \mathbf{h}^{U}=0$. At the history of any sharp interface between different media, given as the piecewise smooth (non-null) spacetime hypersurface $f=0$, the system of Maxwell equations is supplemented by interface conditions on the fields $F$ and $G$

$$
\begin{gathered}
{\left.[F]\right|_{f=0} \wedge d f=0} \\
{\left.[\star G]\right|_{f=0} \wedge d f=j_{s},}
\end{gathered}
$$

\footnotetext{
10 All electromagnetic tensors in this article have dimensions constructed from the SI dimensions $[M],[L],[T],[Q]$ where $[Q]$ has the unit of the Coulomb in this system. We adopt $[g]=\left[L^{2}\right],[G]=[j]=[Q],[F]=\frac{[Q]}{\left[\epsilon_{0}\right]}$ where the permittivity of free space $\epsilon_{0}$ has the dimensions $\left[Q^{2} T^{2} M^{-1} L^{-3}\right]$ and $c_{0}=\frac{1}{\sqrt{\epsilon_{0} \mu_{0}}}$ denotes the speed of light in vacuo. Note that the operators $d$ and $\nabla$ preserve the physical dimensions of tensor fields but with $[g]=\left[L^{2}\right]$, for $p$-forms $\alpha$ in 4 dimensions, one has $[\star \alpha]=[\alpha]\left[L^{4-2 p}\right]$.
} 
where $[H]$ denotes the discontinuity in the field $H$ across the hypersurface. The 3 -form $j_{s}$ on the hypersurface is non zero if it supports a real current 3 -form there.

\section{Time-Dependent Maxwell Systems in 3-Space}

The spacetime description above is natural for the Maxwell system since it makes no reference to any particular frame in spacetime. However to make contact with descriptions in particular frames or non-relativistic formulations a reduction in terms of frame dependent fields becomes mandatory. The spacetime Maxwell system can now be reduced to a family of parameterized exterior systems on $\mathbb{R}^{3}$. Each member is an exterior system involving forms on $\mathbb{R}^{3}$ depending parametrically on some time coordinate $t$ associated with $U$. Let the $(3+1)$ split of the electric 4-current 3 -form with respect to a foliation of spacetime by spacelike hypersurfaces with constant $t$ be

$$
j=\frac{\mathcal{J}^{U}}{c_{0}} \wedge \widetilde{U}+\rho^{U},
$$

with $i_{U} \mathcal{J}^{U}=i_{U} \rho^{U}=0$ and $\rho^{U}=\widehat{\rho}^{U} \# 1$, where $\mathcal{J}^{U}, \rho^{U}$ are the spatial electric current density 2 -form and spatial electric charge density 3 -form respectively. The differential operator $\underline{d}$ on spacetime forms is adapted to those spacetimes (such as Minkowski spacetime where gravity is absent) that can be foliated by hypersurfaces with constant coordinate $t$ where $U=\frac{1}{c_{0}} \partial_{t}$. Then, from (47),

$$
d j=0
$$

yields

$$
\underline{d} \mathcal{J}^{U}+\dot{\rho}^{U}=0 .
$$

The $(3+1)$ split of the spacetime covariant Maxwell equations (47) with respect to $\widetilde{U}=-c_{0} d t$ becomes

$$
\begin{aligned}
\underline{d} \mathbf{e}^{U} & =-\dot{\mathbf{B}}^{U} \\
\underline{d} \mathbf{B}^{U} & =0 \\
\underline{d} \mathbf{h}^{U} & =\mathcal{J}^{U}+\dot{\mathbf{D}}^{U} \\
\underline{d} \mathbf{D}^{U} & =\rho^{U}
\end{aligned}
$$

where $\mathbf{D}^{U}=\# \mathbf{d}^{U}$ and $\mathbf{B}^{U}=\# \mathbf{b}^{U}$. All $p$-forms $(p>0)$ in these equations are independent of $d t$, but have components that may depend parametrically on $t$.

\section{E Electromagnetic Constitutive Relations}

The two 2-forms $F$ and $G$ in the macroscopic Maxwell equations on spacetime are fundamentally related by smoothing the microscopic sources of the electromagnetic fields in the medium. In many circumstances one then relies on 
phenomenological relations for closure relations. In such relations the excitation form $G$ is in general a functional (possibly non-local in spacetime) of the Maxwell form $F$, its covariant differentials, thermodynamic properties, deformation properties and the state of motion of the medium:

$$
G=\mathcal{Z}[F, \nabla F \ldots]
$$

Such a functional may induce non-linear and non-local relations between $\mathbf{d}^{U}, \mathbf{h}^{U}$ and $\mathbf{e}^{U}, \mathbf{b}^{U}$. Electrostriction and magnetostriction arise from the dependence of $\mathcal{Z}$ on the deformation tensor of the medium and its covariant derivatives. For general linear continua, a knowledge of a collection of constitutive tensor fields $Z^{(r)}$ on spacetime may suffice so that

$$
G=\sum_{r=0}^{N} Z^{(r)}\left[\nabla^{r} F, \ldots\right] .
$$

In idealized (non-dispersive) simple continua, one adopts the idealized local relation

$$
G=Z(F),
$$

for some degree 4 constitutive tensor field $Z$, parameterized by scalars that depend on the medium. In the vacuum $G=\epsilon_{0} F$ where $\epsilon_{0}$ is the constant permittivity of the vacuum. Regular lossless, non-conducting, linear isotropic media can be described by a bulk 4 -velocity field $V$ of the medium, a real relative permittivity scalar field $\epsilon_{r}>0$ and a real relative permeability scalar field $\mu_{r}>0$. In this case, the structure of the tensor $Z$ follows from

$$
\begin{aligned}
G & =\epsilon_{0} \epsilon_{r} i_{V} F \wedge \widetilde{V}-\frac{\epsilon_{0}}{\mu_{r}} \star\left(i_{V} \star F \wedge \widetilde{V}\right) \\
& =\epsilon_{0}\left(\epsilon_{r}-\frac{1}{\mu_{r}}\right) i_{V} F \wedge \widetilde{V}+\frac{\epsilon_{0}}{\mu_{r}} F .
\end{aligned}
$$

For inhomogeneous media the relative permittivity and permeability scalars $\epsilon_{r}$ and $\mu_{r}$ will not be constants. In a general frame $U$ comoving with the medium $(U=V)$, (60) yields

$$
\mathbf{d}^{V}=\epsilon_{0} \epsilon_{r} \mathbf{e}^{V} \quad \text { and } \quad \mathbf{h}^{V}=\left(\mu_{0} \mu_{r}\right)^{-1} \mathbf{b}^{V},
$$

which are the familiar closure relations for simple (idealized) electrically neutral isotropic non-dispersive polarizable media.

\section{E.1 Minkowski Constitutive Relations for Moving Media}

The above algebraic constitutive relation (60) involves the bulk 4-velocity field of a simple medium. It is straightforward to find the induced relations between the fields $\left\{\mathbf{e}^{U}, \mathbf{b}^{U}, \mathbf{d}^{U}, \mathbf{h}^{U}\right\}$ relative to an observer in a frame $U \neq V$. From (49) 
and (51), one has

$$
\begin{aligned}
\mathbf{e}^{V} & =i_{V} F=\mathbf{e}^{U}(V) \widetilde{U}-\widetilde{U}(V) \mathbf{e}^{U}-\star\left(c_{0} \mathbf{b}^{U} \wedge \widetilde{U} \wedge \widetilde{V}\right) \\
c_{0} \mathbf{b}^{V} & =i_{V} \star F=\star\left(\mathbf{e}^{U} \wedge \widetilde{U} \wedge \widetilde{V}\right)+c_{0} \mathbf{b}^{U}(V) \widetilde{U}-c_{0} \widetilde{U}(V) \mathbf{b}^{U} \\
\mathbf{d}^{V} & =i_{V} G=\mathbf{d}^{U}(V) \widetilde{U}-\widetilde{U}(V) \mathbf{d}^{U}-\star\left(\frac{\mathbf{h}^{U}}{c_{0}} \wedge \widetilde{U} \wedge \widetilde{V}\right) \\
\frac{\mathbf{h}^{V}}{c_{0}} & =i_{V} \star G=\star\left(\mathbf{d}^{U} \wedge \widetilde{U} \wedge \widetilde{V}\right)+\frac{\mathbf{h}^{U}(V)}{c_{0}} \widetilde{U}-\frac{\widetilde{U}(V)}{c_{0}} \mathbf{h}^{U} .
\end{aligned}
$$

Inserting these in the constitutive relations (61) yields

$$
\begin{aligned}
\mathbf{d}^{U}(V) \widetilde{U}-\widetilde{U}(V) \mathbf{d}^{U}-\star\left(\frac{\mathbf{h}^{U}}{c_{0}} \wedge \widetilde{U} \wedge \widetilde{V}\right) & =\epsilon\left[\mathbf{e}^{U}(V) \widetilde{U}-\widetilde{U}(V) \mathbf{e}^{U}-\star\left(c_{0} \mathbf{b}^{U} \wedge \widetilde{U} \wedge \widetilde{V}\right)\right] \\
c_{0} \star\left(\mathbf{d}^{U} \wedge \widetilde{U} \wedge \widetilde{V}\right)+\mathbf{h}^{U}(V) \widetilde{U}-\widetilde{U}(V) \mathbf{h}^{U} & =\mu^{-1}\left[\star\left(\frac{\mathbf{e}^{U}}{c_{0}} \wedge \widetilde{U} \wedge \widetilde{V}\right)+\mathbf{b}^{U}(V) \widetilde{U}-\widetilde{U}(V) \mathbf{b}^{U}\right],
\end{aligned}
$$

where $\epsilon=\epsilon_{r} \epsilon_{0}, \mu=\mu_{r} \mu_{0}$ in terms of the relative permittivity scalar $\epsilon_{r}$ and relative permeability $\mu_{r}$. Contracting with $U$ gives the relations

$$
\begin{aligned}
\mathbf{d}^{U}(V) & =\epsilon \mathbf{e}^{U}(V) \\
\mathbf{h}^{U}(V) & =\mu^{-1} \mathbf{b}^{U}(V),
\end{aligned}
$$

which yields

$$
\begin{aligned}
& \widetilde{U}(V) \mathbf{d}^{U}+\star\left(\frac{\mathbf{h}^{U}}{c_{0}} \wedge \widetilde{U} \wedge \widetilde{V}\right)=\epsilon\left[\widetilde{U}(V) \mathbf{e}^{U}+\star\left(c \mathbf{b}^{U} \wedge \widetilde{U} \wedge \widetilde{V}\right)\right] \\
& \widetilde{U}(V) \mathbf{b}^{U}-\star\left(\frac{\mathbf{e}^{U}}{c_{0}} \wedge \widetilde{U} \wedge \widetilde{V}\right)=\mu\left[\widetilde{U}(V) \mathbf{h}^{U}-c_{0} \star\left(\mathbf{d}^{U} \wedge \widetilde{U} \wedge \widetilde{V}\right)\right] .
\end{aligned}
$$

A laboratory Minkowski inertial frame is described by $U=\frac{1}{c_{0}} \partial_{t}$ in inertial Cartesian coordinates $\left\{t, x^{1}, x^{2}, x^{3}\right\}$ with $\left\{e^{0}=-c_{0} d t, e^{1}=d x^{1}, e^{2}=d x^{2}, e^{3}=\right.$ $\left.d x^{3}\right\}$ and, relative to $U$, the medium 4 -velocity $V$ has the orthogonal decomposition:

$$
V=\gamma\left(U+\frac{\mathbb{V}^{U}}{c_{0}}\right)
$$

where the spatial field $\mathbb{V}^{U}$ is the Newtonian velocity field 11 of the medium relative to $U, \gamma^{-1} \equiv \sqrt{\left(1-\frac{\nu^{2}}{c_{0}^{2}}\right)}$ and $\nu^{2} \equiv g\left(\mathbb{V}^{U}, \mathbb{V}^{U}\right)$. With these definitions it follows that:

$$
g(U, V)=-\gamma \quad \text { and } \quad \widetilde{U} \wedge \widetilde{V}=\frac{\gamma}{c_{0}} \widetilde{U} \wedge \widetilde{\mathbb{V}}^{U} .
$$

Using these in (63) and rearranging yields

$$
\begin{aligned}
& \mathbf{d}^{U}-\star\left(\widetilde{U} \wedge \widetilde{\mathbb{V}}^{U} \wedge \frac{\mathbf{h}^{U}}{c_{0}^{2}}\right)=\epsilon\left[\mathbf{e}^{U}-\star\left(\widetilde{U} \wedge \widetilde{\mathbb{V}}^{U} \wedge \mathbf{b}^{U}\right)\right] \\
& \mathbf{b}^{U}+\star\left(\widetilde{U} \wedge \widetilde{\mathbb{V}}^{U} \wedge \frac{\mathbf{e}^{U}}{c_{0}^{2}}\right)=\mu\left[\mathbf{h}^{U}+\star\left(\widetilde{U} \wedge \widetilde{\mathbb{V}}^{U} \wedge \mathbf{d}^{U}\right)\right],
\end{aligned}
$$

\footnotetext{
${ }^{11}$ In inertial Cartesian coordinates $\mathbb{V}^{U}=\sum_{j=1}^{3} v^{j}\left(t, x^{1}, x^{2}, x^{3}\right) \frac{\partial}{\partial x^{j}}$.
} 
or using the spatial Hodge map \# defined by $U$ :

$$
\begin{aligned}
& \mathbf{d}^{U}+\#\left(\widetilde{\mathbb{V}}^{U} \wedge \frac{\mathbf{h}^{U}}{c_{0}^{2}}\right)=\epsilon\left[\mathbf{e}^{U}+\#\left(\widetilde{\mathbb{V}}^{U} \wedge \mathbf{b}^{U}\right)\right] \\
& \mathbf{b}^{U}-\#\left(\widetilde{\mathbb{V}}^{U} \wedge \frac{\mathbf{e}^{U}}{c_{0}^{2}}\right)=\mu\left[\mathbf{h}^{U}-\#\left(\widetilde{\mathbb{V}}^{U} \wedge \mathbf{d}^{U}\right)\right] .
\end{aligned}
$$

These are the constitutive relations first written by Minkowski. For some purposes it is useful to decouple these expressions and express $\mathbf{e}^{U}$ and $\mathbf{h}^{U}$ directly in terms of $\mathbf{d}^{U}, \mathbf{b}^{U}$ and $\mathbb{V}^{U}$.

\section{E.2 Minkowski Constitutive Relations for $\mathbf{e}^{U}\left(\mathbf{d}^{U}, \mathbf{b}^{U}, \mathbb{V}^{U}\right), \mathbf{h}^{U}\left(\mathbf{d}^{U}, \mathbf{b}^{U}, \mathbb{V}^{U}\right)$}

Taking the exterior product of (65) with $\mathbb{V}^{U}$ yields,

$$
\mu \widetilde{\mathbb{V}}^{U} \wedge \mathbf{h}^{U}=\widetilde{\mathbb{V}}^{U} \wedge \mathbf{b}^{U}-\widetilde{\mathbb{V}}^{U} \wedge \#\left(\widetilde{\mathbb{V}}^{U} \wedge \frac{\mathbf{e}^{U}}{c_{0}^{2}}\right)+\mu \widetilde{\mathbb{V}}^{U} \wedge \#\left(\widetilde{\mathbb{V}}^{U} \wedge \mathbf{d}^{U}\right) .
$$

For any spatial 1-form $\alpha^{U}$ with respect to $U$ one has:

$$
\begin{aligned}
\widetilde{\mathbb{V}}^{U} \wedge \#\left(\widetilde{\mathbb{V}}^{U} \wedge \alpha^{U}\right) & =-\widetilde{\mathbb{V}}^{U} \wedge \star\left(\widetilde{U} \wedge \widetilde{\mathbb{V}}^{U} \wedge \alpha^{U}\right)=-\star i_{\mathbb{V}}\left(\widetilde{U} \wedge \widetilde{\mathbb{V}}^{U} \wedge \alpha^{U}\right) \\
& =\nu^{2} \star\left(\widetilde{U} \wedge \alpha^{U}\right)-\alpha^{U}\left(\mathbb{V}^{U}\right) \star\left(\widetilde{U} \wedge \widetilde{\mathbb{V}}^{U}\right) \\
& =-\nu^{2} \# \alpha^{U}+\alpha^{U}\left(\mathbb{V}^{U}\right) \# \widetilde{\mathbb{V}}^{U} .
\end{aligned}
$$

Thus

$$
\begin{aligned}
\mu \widetilde{\mathbb{V}}^{U} \wedge \mathbf{h}^{U} & =\widetilde{\mathbb{V}}^{U} \wedge \mathbf{b}^{U}+\frac{\nu^{2}}{c_{0}^{2}} \# \mathbf{e}^{U}-\frac{\mathbf{e}^{U}\left(\mathbb{V}^{U}\right)}{c_{0}^{2}} \# \widetilde{\mathbb{V}}^{U}-\mu \nu^{2} \# \mathbf{d}^{U}+\mu \mathbf{d}^{U}\left(\mathbb{V}^{U}\right) \# \widetilde{\mathbb{V}}^{U} \\
& =\widetilde{\mathbb{V}}^{U} \wedge \mathbf{b}^{U}+\frac{\nu^{2}}{c_{0}^{2}} \# \mathbf{e}^{U}-\mu \nu^{2} \# \mathbf{d}^{U}-\frac{1}{\epsilon c_{0}^{2}}\left(1-\epsilon \mu c_{0}^{2}\right) \mathbf{d}^{U}\left(\mathbb{V}^{U}\right) \# \widetilde{\mathbb{V}}^{U},
\end{aligned}
$$

using the identity $\mathbf{d}^{U}\left(\mathbb{V}^{U}\right)=\epsilon \mathbf{e}^{U}\left(\mathbb{V}^{U}\right)$, obtained by contracting (65) with $\mathbb{V}^{U}$. Since \#\# = 1

$\mu \#\left(\widetilde{\mathbb{V}}^{U} \wedge \mathbf{h}^{U}\right)=\#\left(\widetilde{\mathbb{V}}^{U} \wedge \mathbf{b}^{U}\right)+\frac{\nu^{2}}{c_{0}^{2}} \mathbf{e}^{U}-\mu \nu^{2} \mathbf{d}^{U}-\frac{1}{\epsilon c_{0}^{2}}\left(1-\epsilon \mu c_{0}^{2}\right) \mathbf{d}^{U}\left(\mathbb{V}^{U}\right) \widetilde{\mathbb{V}}^{U}$.

Substituting this into the first relation of (65) yields

$$
\left(\epsilon \mu c_{0}^{2}-\frac{\nu^{2}}{c_{0}^{2}}\right) \mathbf{e}^{U}=\mu c_{0}^{2}\left(1-\frac{\nu^{2}}{c_{0}^{2}}\right) \mathbf{d}^{U}-\left(\epsilon \mu c_{0}^{2}-1\right)\left(\#\left(\widetilde{\mathbb{V}}^{U} \wedge \mathbf{b}^{U}\right)-\frac{\mathbf{d}^{U}\left(\mathbb{V}^{U}\right)}{\epsilon c_{0}^{2}} \widetilde{\mathbb{V}}^{U}\right) .
$$

Since

$$
\frac{1}{\epsilon \mu}=\frac{c_{0}^{2}}{\mathcal{N}^{2}}
$$

where $\mathcal{N}^{2}=\epsilon_{r} \mu_{r}$ is the square of the medium refractive index scalar, this may be written

$$
\left(\mathcal{N}^{2}-\frac{\nu^{2}}{c_{0}^{2}}\right) \mathbf{e}^{U}=\frac{\mathcal{N}^{2}}{\epsilon}\left(1-\frac{\nu^{2}}{c_{0}^{2}}\right) \mathbf{d}^{U}-\left(\mathcal{N}^{2}-1\right)\left(\#\left(\widetilde{\mathbb{V}}^{U} \wedge \mathbf{b}^{U}\right)-\frac{\mathbf{d}^{U}\left(\mathbb{V}^{U}\right)}{\epsilon c_{0}^{2}} \widetilde{\mathbb{V}}^{U}\right) .
$$


Furthermore from (65)

$$
\begin{aligned}
\epsilon \#\left(\widetilde{\mathbb{V}}^{U} \wedge \mathbf{e}^{U}\right) & =\#\left(\widetilde{\mathbb{V}}^{U} \wedge \mathbf{d}^{U}\right)+\left(\frac{\mathbf{h}^{U}\left(\mathbb{V}^{U}\right)}{c_{0}^{2}} \widetilde{\mathbb{V}}^{U}-\frac{\nu^{2}}{c_{0}^{2}} \mathbf{h}^{U}\right)-\epsilon\left(\mathbf{b}^{U}\left(\mathbb{V}^{U}\right) \widetilde{\mathbb{V}}^{U}-\nu^{2} \mathbf{b}^{U}\right) \\
& =\#\left(\widetilde{\mathbb{V}}^{U} \wedge \mathbf{d}^{U}\right)-\frac{\nu^{2}}{c_{0}^{2}} \mathbf{h}^{U}+\epsilon \nu^{2} \mathbf{b}^{U}-\frac{1}{\mu c_{0}^{2}}\left(\epsilon \mu c_{0}^{2}-1\right) \mathbf{b}^{U}\left(\mathbb{V}^{U}\right) \widetilde{\mathbb{V}}^{U},
\end{aligned}
$$

using $\mathbf{b}^{U}\left(\mathbb{V}^{U}\right)=\mu \mathbf{h}^{U}\left(\mathbb{V}^{U}\right)$, obtained by contracting (65) with $\mathbb{V}^{U}$. Substituting this into the second relation of (65) yields

$$
\left(\mathcal{N}^{2}-\frac{\nu^{2}}{c_{0}^{2}}\right) \mathbf{h}^{U}=\frac{\mathcal{N}^{2}}{\mu}\left(1-\frac{\nu^{2}}{c_{0}^{2}}\right) \mathbf{b}^{U}-\left(\mathcal{N}^{2}-1\right)\left(-\#\left(\widetilde{\mathbb{V}}^{U} \wedge \mathbf{d}^{U}\right)-\frac{\mathbf{b}^{U}\left(\mathbb{V}^{U}\right)}{\mu c_{0}^{2}} \widetilde{\mathbb{V}}^{U}\right) .
$$

Thus, the constitutive relations (60) can also be written

$$
\begin{aligned}
& \left(\mathcal{N}^{2}-\frac{\nu^{2}}{c_{0}^{2}}\right) \mathbf{e}^{U}=\frac{\mathcal{N}^{2}}{\epsilon}\left(1-\frac{\nu^{2}}{c_{0}^{2}}\right) \mathbf{d}^{U}+\left(\mathcal{N}^{2}-1\right)\left(-\#\left(\widetilde{\mathbb{V}}^{U} \wedge \mathbf{b}^{U}\right)-\frac{\mathbf{d}^{U}\left(\mathbb{V}^{U}\right)}{\epsilon c_{0}^{2}} \widetilde{\mathbb{V}}^{U}\right) \\
& \left(\mathcal{N}^{2}-\frac{\nu^{2}}{c_{0}^{2}}\right) \mathbf{h}^{U}=\frac{\mathcal{N}^{2}}{\mu}\left(1-\frac{\nu^{2}}{c_{0}^{2}}\right) \mathbf{b}^{U}-\left(\mathcal{N}^{2}-1\right)\left(-\#\left(\widetilde{\mathbb{V}}^{U} \wedge \mathbf{d}^{U}\right)-\frac{\mathbf{b}^{U}\left(\mathbb{V}^{U}\right)}{\mu c_{0}^{2}} \widetilde{\mathbb{V}}^{U}\right) .
\end{aligned}
$$

In the non-relativistic limit (to first order in $\frac{\nu}{c_{0}}$ ) these constitutive relations become

$$
\begin{aligned}
\mathbf{e}^{U} & \approx \frac{\mathbf{d}^{U}}{\epsilon_{0} \epsilon_{r}}-\left(1-\frac{1}{\epsilon_{r} \mu_{r}}\right) \#\left(\widetilde{\mathbb{V}}^{U} \wedge \mathbf{b}^{U}\right) \\
\mathbf{h}^{U} & \approx \frac{\mathbf{b}^{U}}{\mu_{0} \mu_{r}}+\left(1-\frac{1}{\epsilon_{r} \mu_{r}}\right) \#\left(\widetilde{\mathbb{V}}^{U} \wedge \mathbf{d}^{U}\right) .
\end{aligned}
$$

\section{F Polarization and Magnetization}

The polarization 2 -form $\Pi$ in spacetime is defined by

$$
\Pi=G-\epsilon_{0} F \text {. }
$$

The second macroscopic Maxwell equation may then be written

$$
\epsilon_{0} d \star F=j-d \star \Pi=j+j_{p},
$$

where

$$
j_{p}=-d \star \Pi
$$

will be called the electric polarization current 3 -form. With respect to any observer frame $U$ its orthogonal decomposition is

$$
\Pi=\mathbf{p}^{U} \wedge \widetilde{U}-\star\left(\frac{\mathbf{m}^{U}}{c_{0}} \wedge \widetilde{U}\right)=\mathbf{p}^{U} \wedge \widetilde{U}-\frac{1}{c_{0}} \mathbf{M}^{U},
$$

where $\mathbf{M}^{U}=\# \mathbf{m}^{U}$ and we call

$$
\mathbf{p}^{U}=i_{U} \Pi \quad \text { and } \quad \frac{\mathbf{m}^{U}}{c_{0}}=i_{U} \star \Pi
$$


the spatial polarization 1-form and magnetization 1-form respectively relative to $U$. The Hodge dual of $\Pi$ has the decomposition

$$
\star \Pi=\star\left(\mathbf{p}^{U} \wedge \widetilde{U}\right)+\frac{\mathbf{m}^{U}}{c_{0}} \wedge \widetilde{U}=\mathbf{P}^{U}+\frac{1}{c_{0}} \mathbf{m}^{U} \wedge \widetilde{U},
$$

where $\mathbf{P}^{U}=\# \mathbf{p}^{U}$. From (51), (49), (68) and (70) it follows

$$
\mathbf{d}^{U}=\epsilon_{0} \mathbf{e}^{U}+\mathbf{p}^{U} \quad \text { and } \quad \mathbf{h}=\mu_{0}^{-1} \mathbf{b}^{U}+\mathbf{m}^{U} .
$$

From (69), (70) and (42) one finds

$$
\begin{aligned}
j_{p} & =-d \mathbf{P}^{U}-\frac{d \mathbf{m}^{U}}{c_{0}} \wedge \widetilde{U}=-\underline{d} \mathbf{P}^{U}+\widetilde{U} \wedge \mathcal{L}_{U} \mathbf{P}^{U}-\frac{d \mathbf{m}^{U}}{c_{0}} \wedge \widetilde{U} \\
& =-\underline{d} \mathbf{P}^{U}+\frac{1}{c_{0}}\left(c_{0} \mathcal{L}_{U} \mathbf{P}^{U}-\underline{d} \mathbf{m}^{U}\right) \wedge \widetilde{U} \\
& =-\underline{d} \mathbf{P}^{U}+\frac{1}{c_{0}}\left(\dot{\mathbf{P}}^{U}-\underline{d} \mathbf{m}^{U}\right) \wedge \widetilde{U} .
\end{aligned}
$$

Writing the orthogonal decomposition of $j_{p}$ with respect to $U$ as

$$
j_{p}=\frac{\mathcal{J}_{p}^{U}}{c_{0}} \wedge \widetilde{U}+\rho_{p}^{U},
$$

it follows that

$$
\frac{\mathcal{J}_{p}^{U}}{c_{0}}=-i_{U} j_{p}=\frac{1}{c_{0}}\left(\dot{\mathbf{P}}^{U}-\underline{d} \mathbf{m}^{U}\right) \quad \text { and } \quad \rho_{p}^{U}=-\left(i_{U} \star j_{p}\right) \star \widetilde{U}=-\underline{d} \mathbf{P}^{U} .
$$

In the frame $U, \mathcal{J}_{p}^{U}$ and $\rho_{p}^{U}$ denote the induced electric polarization current density spatial 2 -form and induced polarization charge density spatial 3-form respectively. In a similar manner

$$
\begin{aligned}
d \Pi & =d \mathbf{p}^{U} \wedge \widetilde{U}-\frac{1}{c_{0}} d \mathbf{M}^{U}=\underline{d} \mathbf{p}^{U} \wedge \widetilde{U}-\frac{1}{c_{0}} \underline{d} \mathbf{M}^{U}+\frac{1}{c_{0}} \widetilde{U} \wedge \mathcal{L}_{U} \mathbf{M}^{U} \\
& =\frac{1}{c_{0}}\left(c_{0} \underline{d} \mathbf{p}^{U}+\mathcal{L}_{U} \mathbf{M}^{U}\right) \wedge \widetilde{U}-\frac{1}{c_{0}} \underline{d} \mathbf{M}^{U} .
\end{aligned}
$$

with the orthogonal decomposition $j_{m}=d \Pi=\frac{\mathcal{J}_{m}^{U}}{c_{0}} \wedge \widetilde{U}+\rho_{m}^{U}$ where

$\frac{\mathcal{J}_{m}^{U}}{c_{0}}=-i_{U} j_{m}=\frac{1}{c_{0}}\left(c_{0} \underline{d} \mathbf{p}^{U}+\frac{1}{c_{0}} \dot{\mathbf{M}}^{U}\right) \quad$ and $\quad \rho_{m}^{U}=-\left(i_{U} \star j_{m}\right) \star \widetilde{U}=-\frac{1}{c_{0}} \underline{d} \mathbf{M}^{U}$

denote the induced magnetization charge current density spatial 2-form and induced magnetization charge density spatial 3 -form respectively in terms of $\mathbf{p}^{U}$ and $\mathbf{M}^{U}$.

\section{G Derivation of the Model Force Density 3-form}

The key steps in deriving the expression for the force density 3-form in $\S 2$ of paper I are outlined (equation 2.14), with the aid of the identities

$$
\begin{aligned}
\# \alpha \wedge \beta & =\# i_{\widetilde{\beta}} \alpha, \\
\mathcal{L}_{\widetilde{\beta}} \alpha & =i_{\widetilde{\beta}} \underline{d} \alpha+\underline{d} i_{\widetilde{\beta}} \alpha \quad \text { (Cartan's identity) } \\
\underline{d}(\alpha \wedge \# \beta) & =0
\end{aligned}
$$


for any spatial 1-forms $\alpha, \beta$. From equation (2.8) of $\S 2$, paper I, we have

$$
\mathcal{F}_{K}=\frac{1}{2}\left[\underline{d}\left(\mathbf{e}^{U} \cdot \mathbf{p}^{U}+\mathbf{b}^{U} \cdot \mathbf{m}^{U}\right)+\# \mathcal{L}_{\partial_{t}}\left(\mathbf{p}^{U} \wedge \mathbf{b}^{U}\right)\right] \wedge \kappa,
$$

with $\kappa=\# \widetilde{K}$. The first term may be expressed

$$
\begin{aligned}
\underline{d}\left(\mathbf{e}^{U} \cdot \mathbf{p}^{U}\right) & =\underline{d} i_{\widetilde{p^{U}}} \mathbf{e}^{U}=\mathcal{L}_{\widetilde{\mathbf{p}^{U}}} \mathbf{e}^{U}-i_{\widetilde{\mathbf{p}^{U}}} \underline{d} \mathbf{e}^{U} \\
& =\mathcal{L}_{\widetilde{\mathbf{p}^{U}}} \mathbf{e}^{U}-\# \# i_{\mathbf{p}^{U}} \underline{d} \mathbf{e}^{U}=\mathcal{L}_{\mathbf{p}^{U}} \mathbf{e}^{U}-\#\left(\# \underline{d} \mathbf{e}^{U} \wedge \mathbf{p}^{U}\right),
\end{aligned}
$$

using (73) and (74). The last term can be written

$$
\begin{aligned}
\# \mathcal{L}_{\partial_{t}}\left(\mathbf{p}^{U} \wedge \mathbf{b}^{U}\right) & =\#\left(\dot{\mathbf{p}}^{U} \wedge \mathbf{b}^{U}+\mathbf{p}^{U} \wedge \dot{\mathbf{b}}^{U}\right)=\#\left(\dot{\mathbf{p}}^{U} \wedge \mathbf{b}^{U}-\mathbf{p}^{U} \wedge \# \underline{d} \mathbf{e}^{U}\right) \\
& =\#\left(\dot{\mathbf{p}}^{U} \wedge \mathbf{b}^{U}\right)+\#\left(\# \underline{d} \mathbf{e}^{U} \wedge \mathbf{p}^{U}\right),
\end{aligned}
$$

using (56). Finally, the second term is

$$
\begin{aligned}
\underline{d}\left(\mathbf{b}^{U} \cdot \mathbf{m}^{U}\right) & =\underline{d} i_{\widetilde{b^{U}}} \mathbf{m}^{U}=\mathcal{L}_{\widetilde{\mathbf{b}^{U}}} \mathbf{m}^{U}-i_{\widetilde{\mathbf{b}^{U}}} \underline{d \mathbf{m}^{U}} \\
& =\mathcal{L}_{\widetilde{\mathbf{b}^{U}}} \mathbf{m}^{U}-\# \# i_{\widetilde{\mathbf{b}^{U}}} \underline{d} \mathbf{m}^{U}=\mathcal{L}_{\widetilde{\mathbf{b}^{U}}} \mathbf{m}^{U}-\#\left(\# \underline{d} \mathbf{m}^{U} \wedge \mathbf{b}^{U}\right),
\end{aligned}
$$

using (73) and (74). From these expressions

$$
\begin{aligned}
2 \mathcal{F}_{K} & =\left[\mathcal{L}_{\widetilde{\mathbf{p}^{U}}} \mathbf{e}^{U}+\mathcal{L}_{\widetilde{\mathbf{b}^{U}}} \mathbf{m}^{U}+\#\left(\left(\dot{\mathbf{p}}^{U}-\# \underline{d \mathbf{m}^{U}}\right) \wedge \mathbf{b}^{U}\right)\right] \wedge \kappa \\
& =\left[\mathcal{L}_{\widetilde{\mathbf{p}^{U}}} \mathbf{e}^{U}+\mathcal{L}_{\widetilde{\mathbf{b}^{U}}} \mathbf{m}^{U}+\#\left(\# \mathcal{J}_{p}^{U} \wedge \mathbf{b}^{U}\right)\right] \wedge \kappa \\
& =\left[\mathcal{L}_{\widetilde{\mathbf{p}^{U}}} \mathbf{e}^{U}+\mathcal{L}_{\widetilde{\mathbf{b}^{U}}} \mathbf{m}^{U}+i_{\widetilde{\mathbf{b}^{U}}} \mathcal{J}_{p}^{U}\right] \wedge \kappa,
\end{aligned}
$$

using (73) and (73). Using Cartan's identity in the first term here gives

$$
\begin{aligned}
\mathcal{L}_{\mathbf{p}^{U}} \mathbf{e}^{U} \wedge \kappa & =\mathcal{L}_{\widetilde{p^{U}}}\left(\mathbf{e}^{U} \wedge \kappa\right)-\mathbf{e}^{U} \wedge \mathcal{L}_{\widetilde{\mathbf{p}^{U}}} \kappa \\
& =\underline{d} i_{\widetilde{p^{U}}}\left(\mathbf{e}^{U} \wedge \kappa\right)-\mathbf{e}^{U} \wedge \underline{d} i_{\widetilde{\mathbf{p}^{U}}} \kappa-\mathbf{e}^{U} \wedge i_{\widetilde{\mathbf{p}^{U}}} \underline{d} \kappa \\
& =\underline{d} i_{\widetilde{p^{U}}}\left(\mathbf{e}^{U}(K) \# 1\right)-\mathbf{e}^{U} \wedge \underline{d} \#\left(\widetilde{K} \wedge \mathbf{p}^{U}\right) \\
& =\underline{d}\left(\mathbf{e}^{U}(K) \mathbf{P}^{U}\right)+\mathbf{e}^{U} \wedge \underline{d} \#\left(\mathbf{p}^{U} \wedge \widetilde{K}\right),
\end{aligned}
$$

using (74) and (75) and the fact that $K=\frac{\partial}{\partial x^{2}}$ and so $d \kappa=0$. In the last term one has

$$
\begin{aligned}
& \mathbf{e}^{U} \wedge \underline{d} \#\left(\mathbf{p}^{U} \wedge \widetilde{K}\right)=\mathbf{e}^{U} \wedge \underline{d} i_{K} \mathbf{P}^{U}=\mathbf{e}^{U} \wedge \mathcal{L}_{K} \mathbf{P}^{U}-\mathbf{e}^{U} \wedge i_{K} \underline{d} \mathbf{P}^{U} \\
& =\mathbf{e}^{U} \wedge \# \mathcal{L}_{K} \mathbf{p}^{U}-\mathbf{e}^{U}(K) \underline{d} \mathbf{P}^{U} \\
& =\mathcal{L}_{K} \mathbf{p}^{U} \wedge \mathbf{E}^{U}+\rho_{p}^{U} \mathbf{e}^{U}(K),
\end{aligned}
$$

since $K$ is Killing and using (73). This yields

$$
\mathcal{L}_{\widetilde{\mathbf{p}}} \mathbf{e}^{U} \wedge \kappa=\rho_{p}^{U} \mathbf{e}^{U}(K)+\mathcal{L}_{K} \mathbf{p}^{U} \wedge \mathbf{E}^{U}+\underline{d}\left(\mathbf{e}^{U}(K) \mathbf{P}^{U}\right)
$$

and so the force drive 3 -form becomes

$$
\mathcal{F}_{K}=\frac{1}{2}\left[\rho_{p}^{U} \mathbf{e}^{U}(K)+i_{\widetilde{\mathbf{b}^{U}}} \mathcal{J}_{p}^{U} \wedge \kappa+\mathcal{L}_{K} \mathbf{p}^{U} \wedge \mathbf{E}^{U}+\mathcal{L}_{\widetilde{\mathbf{b}^{U}}} \mathbf{m}^{U} \wedge \kappa+\underline{d}\left(\mathbf{e}^{U}(K) \mathbf{P}^{U}\right)\right] .
$$




\section{H Orthogonal Decompositions used in Deriving the Drive 3-form Associated with the Sym- metrized Minkowski Electromagnetic Stress- Energy-Momentum Tensor}

In this section, the key steps used in deriving equation (4.6) of $\S 4$, paper I are outlined. For $\widetilde{U}(K)=0$, one calculates

$$
\begin{aligned}
& \frac{\Pi}{\epsilon_{0}} \wedge i_{K} j=\Pi \wedge\left(\frac{1}{c_{0}} i_{K} \mathcal{J}^{U} \wedge \widetilde{U}+i_{K} \rho^{U}\right) \\
& =\frac{1}{\epsilon_{0}}\left(\mathbf{p}^{U} \wedge \widetilde{U}-\frac{1}{c_{0}} \mathbf{M}^{U}\right) \wedge\left(\frac{1}{c_{0}} i_{K} \mathcal{J}^{U} \wedge \widetilde{U}+i_{K} \rho^{U}\right) \\
& =\frac{1}{\epsilon_{0}} \mathbf{p}^{U} \wedge \widetilde{U} \wedge i_{K} \rho^{U}-\frac{1}{\epsilon_{0} c_{0}^{2}} \mathbf{M}^{U} \wedge i_{K} \mathcal{J}^{U} \wedge \widetilde{U}-\frac{1}{\epsilon_{0} c_{0}} \mathbf{M}^{U} \wedge i_{K} \rho^{U} \\
& =\left(\frac{1}{\epsilon_{0}} \mathbf{p}^{U} \wedge i_{K} \rho^{U}-\mu_{0} \mathbf{M}^{U} \wedge i_{K} \mathcal{J}^{U}\right) \wedge \widetilde{U}-\frac{1}{\epsilon_{0} c_{0}} \mathbf{M}^{U} \wedge i_{K} \rho^{U} \\
& =\left(\frac{1}{\epsilon_{0}} \mathbf{p}^{U}(K) \rho^{U}+\mu_{0} i_{\mathbf{m}^{U}} \mathcal{J}^{U} \wedge \kappa\right) \wedge \widetilde{U}-\frac{1}{\epsilon_{0} c_{0}} \mathbf{M}^{U} \wedge i_{K} \rho^{U} \\
& F \wedge i_{K} j=\left(\mathbf{e}^{U} \wedge \widetilde{U}-c_{0} \mathbf{B}^{U}\right) \wedge\left(\frac{1}{c_{0}} i_{K} \mathcal{J}^{U} \wedge \widetilde{U}+i_{K} \rho^{U}\right) \\
& =\mathbf{e}^{U} \wedge \tilde{U} \wedge i_{K} \rho^{U}-\mathbf{B}^{U} \wedge i_{K} \mathcal{J}^{U} \wedge \widetilde{U}-c_{0} \mathbf{B}^{U} \wedge i_{K} \rho^{U} \\
& =\left(\mathbf{e}^{U} \wedge i_{K} \rho^{U}-\mathbf{B}^{U} \wedge i_{K} \mathcal{J}^{U}\right) \wedge \widetilde{U}-c_{0} \mathbf{B}^{U} \wedge i_{K} \rho^{U} \\
& =\left(\mathbf{e}^{U}(K) \rho^{U}+i_{\widetilde{\mathbf{b}^{U}}} \mathcal{J}^{U} \wedge \kappa\right) \wedge \widetilde{U}-c_{0} \mathbf{B}^{U} \wedge i_{K} \rho^{U} \\
& F \wedge \star i_{K} d \Pi=i_{K} d \Pi \wedge \star F=\left(\frac{1}{c_{0}} i_{K} \mathcal{J}_{m}^{U} \wedge \widetilde{U}+i_{K} \rho_{m}^{U}\right) \wedge\left(\mathbf{E}^{U}+c_{0} \mathbf{b}^{U} \wedge \widetilde{U}\right) \\
& =\frac{1}{c_{0}} i_{K} \mathcal{J}_{m}^{U} \wedge \widetilde{U} \wedge \mathbf{E}^{U}+i_{K} \rho_{m}^{U} \wedge \mathbf{E}^{U}+c_{0} i_{K} \rho_{m}^{U} \wedge \mathbf{b}^{U} \wedge \widetilde{U} \\
& =\left(\frac{1}{c_{0}} i_{K} \mathcal{J}_{m}^{U} \wedge \mathbf{E}^{U}+c_{0} i_{K} \rho_{m}^{U} \wedge \mathbf{b}^{U}\right) \wedge \widetilde{U}+i_{K} \rho_{m}^{U} \wedge \mathbf{E}^{U} \\
& =\left(-\frac{1}{c_{0}} i_{\mathbf{e}^{U}} \mathcal{J}_{m}^{U} \wedge \kappa+c_{0} \rho_{m}^{U} \mathbf{b}^{U}(K)\right) \wedge \widetilde{U}+i_{K} \rho_{m}^{U} \wedge \mathbf{E}^{U} \\
& G \wedge i_{K} d \star \frac{\Pi}{\epsilon_{0}}=-\frac{1}{\epsilon_{0}} i_{K} G \wedge d \star \Pi=-i_{K} F \wedge d \star \Pi-i_{K} \Pi \wedge d \star \frac{\Pi}{\epsilon_{0}} \\
& -i_{K} F \wedge d \star \Pi=-\left(i_{K} \mathbf{e}^{U} \wedge \widetilde{U}-c_{0} i_{K} \mathbf{B}^{U}\right) \wedge\left(-\frac{1}{c_{0}} \mathcal{J}_{p}^{U} \wedge \widetilde{U}-\rho_{p}^{U}\right) \\
& =i_{K} \mathbf{e}^{U} \wedge \widetilde{U} \wedge \rho_{p}^{U}-i_{K} \mathbf{B}^{U} \wedge \mathcal{J}_{p}^{U} \wedge \widetilde{U}-c_{0} i_{K} \mathbf{B}^{U} \wedge \rho_{p}^{U} \\
& =-\left(\mathbf{e}^{U}(K) \rho_{p}^{U}+i_{K} \mathbf{B}^{U} \wedge \mathcal{J}_{p}^{U}\right) \wedge \widetilde{U}-c_{0} i_{K} \mathbf{B}^{U} \wedge \rho_{p}^{U} \\
& =\left(-\mathbf{e}^{U}(K) \rho_{p}^{U}-i_{\widetilde{\mathbf{b}^{U}}} \mathcal{J}_{p}^{U} \wedge \kappa\right) \wedge \widetilde{U}-c_{0} i_{K} \mathbf{B}^{U} \wedge \rho_{p}^{U}
\end{aligned}
$$




$$
\begin{aligned}
-i_{K} \Pi \wedge d \star \frac{\Pi}{\epsilon_{0}} & =-\frac{1}{\epsilon_{0}}\left(i_{K} \mathbf{p}^{U} \wedge \widetilde{U}-\frac{1}{c_{0}} i_{K} \mathbf{M}^{U}\right) \wedge\left(-\frac{1}{c_{0}} \mathcal{J}_{p}^{U} \wedge \widetilde{U}-\rho_{p}^{U}\right) \\
& =\frac{1}{\epsilon_{0}} i_{K} \mathbf{p}^{U} \wedge \widetilde{U} \wedge \rho_{p}^{U}-\frac{1}{\epsilon_{0} c_{0}^{2}} i_{K} \mathbf{M}^{U} \wedge \mathcal{J}_{p}^{U} \wedge \widetilde{U}-\frac{1}{\epsilon_{0} c_{0}} i_{K} \mathbf{M}^{U} \wedge \rho_{p}^{U} \\
& =-\left(\frac{1}{\epsilon_{0}} \mathbf{p}^{U}(K) \rho_{p}^{U}+\mu_{0} i_{K} \mathbf{M}^{U} \wedge \mathcal{J}_{p}^{U}\right) \wedge \widetilde{U}-\frac{1}{\epsilon_{0} c_{0}} i_{K} \mathbf{M}^{U} \wedge \rho_{p}^{U} \\
& =\left(-\frac{1}{\epsilon_{0}} \mathbf{p}^{U}(K) \rho_{p}^{U}-\mu_{0} i_{\mathbf{m}^{U}} \mathcal{J}_{p}^{U} \wedge \kappa\right) \wedge \widetilde{U}-\frac{1}{\epsilon_{0} c_{0}} i_{K} \mathbf{M}^{U} \wedge \rho_{p}^{U} .
\end{aligned}
$$

These expressions may be combined to produce the required 4-form $d \tau_{K}^{S M}$ as described in $\S 4$, paper I.

\section{Bibliography}

[1] A. Kovetz, Electromagnetic Theory, Oxford University Press, (2000)

[2] G.A. Maugin, On the Covariant Equations of the Relativistic Electrodynamics of Continua. III. Elastic Solids, J. Math. Phys., 19 (1978) 12121219

[3] L. D. Landau, E. M. Lifschitz and L. P. Pitaevskit, Electrodynamics of Continuous Media - Volume 8 in Course of Theoretical Physics, (Butterworth-Heinemann, Oxford) 1984

[4] G.A. Maugin, Further Comments on the Equivalence of Abraham's, Minkowski's and Others' Electrodynamics, Can. J. Phys., 58 (1980) 11631170

[5] V. L. GinzBerg, The Laws of Conservation of Energy and Momentum in Emission of Electromagnetic Waves (Photons) in a Medium and the Energy-Momentum Tensor in Macroscopic Electrodynamics, Sov. Phys.Usp., 16 (1973) 434

[6] W. IsRael, Relativistic Effects in Dielectrics: An Experimental Decision between Abraham and Minkowski?, Phys. Letts. B, 67 (1977) 125-127

[7] R. N. C. Pfeifer, T. A. Nieminen, N. R. Heckenberg and H. Rubinsztein-Dunlop, Colloquium: Momentum of an Electromagnetic Wave in Dielectric Media, Rev. Mod. Phys., 79 (2007) 1197-1216

[8] J. Frauendiener and A. Kabobel, The Static Spherically Symmetric Body in Relativistic Elasticity, Class. Quant. Grav., 24 (2007) 4817-4837

[9] E. A. Hinds and S. M. Barnett, Momentum Exchange Between Light and a Single Atom: Abraham or Minkowski?, Phys. Rev. Lett., 102 (2009) 050403:1-4

[10] I. Brevik and A Ellingsen, Transverse Radiation Force in a Tailored Optical Fibre, arXiv:0012.2247v1, (2009)

[11] Y. N. Obukhov and F. W. HehL, Forces and Momenta Caused by Electromagnetic Waves in magneto-electric Media, Phys. Lett. A, 372 (2008) 3946-3952 
[12] Y. N. OBukhov and F. W. Hehl, Electromagnetic Energy-Momentum and Forces in Matter, Phys. Letts. A, 311 (2003) 277-284

[13] G.B. Walker and G. Walker, Mechanical Forces in a Dielectric Due to Electromagnetic Fields, Can. J. Phys., 55 (1977) 2121

[14] G.B. Walker, D.G.Lahoz and G. Walker, Measurement of the Abraham Force in a Barium Titanate Specimen, Can. J. Phys., 53 (1975) 2577

[15] I. BREVIK, Electromagnetic Energy-Momentum Tensor Within Material Media, Mat. Fys. Medd. dan. Vid. Selsk., 37 (1970) 1-11

[16] S. M. Barnett and R. Loudon, On the Electromagnetic Force on a Dielectric Medium, J. Phys. B: At. Mol. Opt. Phys., 39 (2006) 671-684

[17] M. Mansuripur, Electromagnetic Force and Torque in Ponderable Media, Optics Express, 16 (2008) 14821-14835

[18] I. M. Benn and R. W. Tucker, An Introduction to Spinors and Geometry with Applications in Physics, (Adam Hilger: IoP Publishing, Bristol) 1988

[19] R. W. Tucker and T. J. Walton, An Intrinsic Approach to Forces in Magnetoelectric Media, Il Nuovo Cimento C, 32 (2009) 205-229

[20] S. R. DE Groot and L G SutTorp, Foundations of Electrodynamics, North Holland, (1972)

[21] A.I. Murdoch and R. Bedeaux, Continuum Equations of Balance via Weighted Averages of Microscopic Quantities, Proc. Roy. Soc. A, 445 (1994) 1571-179

[22] J. Schwinger, L. L. Deraad, K. A. Milton, Wu-Yang Tsai and J. Norton, Classical Electrodynamics, Perseus Books, (1998)

[23] R. W. Tucker, Differential Form Valued Forms and Distributional Electromagnetic Sources, J. Math. Phys., 50 (2009) 033506:1-28

[24] R. W. TuCKER, A Distributional Formulation of Electromagnetic Fields and Sources in the Presence of Media Discontinuities, J. Phys. (Conference Series) (To Appear)

[25] I. M. Benn, Conservation Laws in Arbitrary Space-times, Ann. Inst. H. Poincaré, 37 (1982) 67-91

[26] H. Minkowski, Die Grundleichungen fur die Elektromagnetischen Vorgange in bewegten Koerpern, Nachr. Ges. Wiss. Gottingen, 53 (1908) 111

[27] M. Abraham, Zur Elektrodynamik bewegter Körper, Rend. Cir. Mat. Palermo, 28 (1909) 1-28

[28] T. Dereli, J. Gratus and R. W. Tucker, The Covariant Description of Electromagnetically Polarizable Media, Phys. Lett. A, 361 (2006) 190-193

[29] T. Dereli, J. Gratus and R.W. Tucker, New Perspectives on the Relevance of Gravitation for the Covariant Description of Electromagnetically Polarizable Media, J. Phys A: Math. Theor., 10 (2007) 5695-5715 